\title{
Idealization of rough sets and medical applications in decision-making the impact factors of COVID-19 infections and heart attacks
}

Mona Hosny ( $\square$ monahosny@edu.asu.edu.eg)

Ain Shams University Faculty of Education https://orcid.org/0000-0003-4258-5257

\section{Research Article}

Keywords: Rough sets, ideals, maximal right neighborhoods, approximations spaces

Posted Date: February 1st, 2022

DOI: https://doi.org/10.21203/rs.3.rs-1159490/v1

License: (c) (1) This work is licensed under a Creative Commons Attribution 4.0 International License.

Read Full License 


\title{
Idealization of rough sets and medical applications in decision-making the impact factors of COVID-19 infections and heart attacks
}

\author{
Mona Hosny ${ }^{1,2 *}$ \\ ${ }^{1}$ Mathematics Department, Faculty of Science, King Khalid University, Abha, Saudi Arabia \\ ${ }^{2}$ Mathematics Department, Faculty of Education, Ain Shams University, Cairo, Egypt
}

\begin{abstract}
Rough set theory is a mathematical technique to address the issues of uncertainty and vagueness in knowledge. It relies mainly on two approximations namely, lower and upper approximations which are used to study the boundary region and accuracy measure. Ideal is considered as a crucial research of extension this theory. As, it is efficacious tool to dispose of vagueness and uncertainties by helping us to approximate the rough set in a more general manner, accurate and increase the accuracy measure. Minimizing the boundary region is one of the pivotal and substantial themes for studying the rough sets which consequently aims to maximize the accuracy measure. Ideal is one of the effective and successful followed methods to achieve this goal perfectly. So, the object through this work is to present new methods in rough set via ideals. Moreover, these methods are also based on the maximal right neighborhood generated by binary relations not similarity relations as in the previous studies. Accordingly, the present techniques are extended the applications fields of the rough sets. As, the similarity relations do not always hold in many real-life applications and consequently this restriction limits the wide applications of this set as it is shown in the end of this paper. Some important characteristics of these methods are scrutinized and demonstrated that they are generated accuracy measures greater and higher than the former ones in the other approaches. Afterwards, it is elucidated that the corresponding upper and lower approximations, boundary regions, accuracy measures and roughness measures are monotonic. Finally, two medical applications are introduced to show the significance of utilizing the ideals in the proposed methods and how it plays an intrinsical and a substantial role in the decision making problems. It enables us to decide the impact factors of COVID-19 infections and heart attacks. As, it handles any imperfect data in symptoms of the diseases and this automatically makes the diagnosis of patients easily and perfectly. Consequently, this help the medical staff to make a precise decision about the diagnosis of patients.
\end{abstract}

Keywords: Rough sets, ideals, maximal right neighborhoods, approximations spaces.

\footnotetext{
*maly@kku.edu.sa,monahosny@edu.asu.edu.eg
} 


\section{Introduction}

The problems of imprecision, ambiguous and incompleteness of information system in data analysis occupied the human mind for long times. These problems exist in different fields such as economics, engineering, medical science, sociality and environmental sciences. Over the years, mathematicians, engineers and scientists, particularly those who focus on artificial intelligence are seeking for powerful tools to solve these problems. They suggested many techniques for this aim such as the rough set theory $[24,25]$ which is the most popular of all these. It deals with uncertainty models by using the concept of lower and upper approximations. If the lower and upper approximations of the set are equal to each other, then it is called a crisp set, otherwise known as a rough set. Therefore, the boundary region is defined as the difference between the upper and lower approximations. This boundary is usually associated with vagueness (i.e., existing of objects which cannot be uniquely classified to the set or its complement). Hence, the accuracy of the set or ambiguous is depending on the boundary region is empty or not respectively. Nonempty boundary region of a set means that our knowledge about the set is not sufficient to define the set precisely. So, the most important goal of the rough set theory is to reduce the boundary region and increase the accuracy of the set. The classical rough approximations are based on an equivalence relation on a finite universe, but this relation is sometimes difficult to be obtained in real-world problems. Extensions of this theory were therefore studied by many approaches in order to deal with complex practical problems. One approach was substituted the equivalence relations by tolerance or similarity relations $[9,10,17,21,23,27,28,29,30]$ or binary relations $[2,3,19,26,34,35,36,37,38]$.

Neighborhoods systems are the most fruitful technique to generalize the rough set theory by replacing an equivalence class with a neighborhood when defining approximations. Several types of neighborhoods were utilized to define the lower and upper approximations such as right and left neighborhoods [32, 33], minimal right neighborhoods [4] and minimal left neighborhoods [5]. Meanwhile, Abo-Tabl [1] defined the approximations by minimal right neighborhoods which determined by reflexive relations that form the base of topological space. More recently, Dai et al. [8] presented three new types of approximations based on the maximal neighborhoods which determined by similarity relations. The thing that distinguished Dai et al.'s approximations [8] from Abo-Tabl's approximations [1] was that the corresponding upper and lower approximations, boundary regions, accuracy measures and roughness measures in two types of Dai et al.'s approximations [8] had the monotonicity. Therefore, Dai et al.'s approximations [8] were considered as improvement of Abo-Tabl's approximations [1].

An ideal is a non-empty collection of sets which is closed under hereditary property and the finite additivity $[22,31]$. The interest in the idealized version of many rough sets models is grown drastically in the past 10 years. The advantage and benefit of using the ideal in this theory is that it reduces the vagueness (uncertainty) of a concept to uncertainty areas at their borders by increasing the lower approximations and decreasing the upper approximations. Consequently, it minimizes the boundary region and improves the accuracy measure. So, ideal is a powerful method 
to demystify from the concept and define it precisely. Accordingly, the study of this theory via ideals is an enjoyable topic that received the attention of many researchers (see: $[6,12,13,14,15$, $18,20])$. Therefore, ideal has been extensively applied in this theory.

The present work is focused on expressing the main concepts of rough set via ideals and maximal neighborhood deduced by binary relations to handle more real applications. This paper comprises of eight sections and its sequence is as follows. After the introduction. Section 2 outlines the necessary concepts and preliminaries which required in the sequel to this work. The purpose of Sections 3, 4, 5 and 6 is to construct four methods to approximate the set by using the notion of ideals and maximal right neighborhoods which induced by binary relations. The properties of the current approximations are interjected and analyzed. It is proved that the boundary of a subset decreases and the accuracy increases as the ideal increases (see Theorems 3.1, 4.1, 5.1 and 6.1). Moreover, it is shown that the corresponding upper and lower approximations, boundary regions, accuracy measures and roughness measures of three types of these approximations are monotonic (see Theorems 3.2, 4.2 and 6.2). Furthermore, the relationships among these approximations are interpreted and illustrated. Additionally, Theorems 3.3, 4.4, 5.3 and 6.4 explain that the present methods reduces the boundary region by increasing the lower approximations and decreasing the upper approximations with the comparison of Dai et al.'s methods [8]. They also show that the current accuracy is greater than the previous one in [8]. Hence, this work is generalization and improvement of Dai et al.'s work [8]. It is worthwhile to note that in the current approximations if the ideal equals to the empty set and the binary relation is a similarity relation, then these approximations coincide with Dai et al.'s approximations [8]. So, Dai et al.'s approximations [8] are special case of the current approximations. In the end, medical applications are presented to illustrate the importance of the present approaches in decision making of two real life applications. It is pointed out that the current techniques enable us to classify patients successfully in terms of infection or not with both of the new corona-virus COVID-19 (first application) and heart attacks (second application). In the first application, a similarity relation is used while in the second application a binary relation is applied. Therefore, Dai et al.'s methods [8] can not be applied in the second application. As, they based on the similarity relations and this restricts the applications of the rough set theory. It emphasizes that the present methods are open the way for more applications. Eventually, the conclusion of this paper and remarks for future research work are discussed in Section 8.

\section{Preliminaries}

Before proceeding further, let me first recall some fundamentals concepts which are beneficial and required in the sequel of this work.

Definition 2.1. [16] A non-empty collection $\mathcal{I}$ of subsets of a set $U$ is called an ideal on $U$, if it satisfies the following conditions

$$
\text { 1. } A \in \mathcal{I} \text { and } B \in \mathcal{I} \Rightarrow A \cup B \in \mathcal{I} \text {, }
$$


2. $A \in \mathcal{I}$ and $B \subseteq A \Rightarrow B \in \mathcal{I}$.

i.e., $\mathcal{I}$ is closed under finite unions and subsets.

Definition 2.2. [18] Let $\mathcal{I}_{1}, \mathcal{I}_{2}$ be two ideals on a non-empty set $U$. The smallest collection generating by $\mathcal{I}_{1}, \mathcal{I}_{2}$ is denoted by $\mathcal{I}_{1} \vee \mathcal{I}_{2}$ and defined as:

$$
\mathcal{I}_{1} \vee \mathcal{I}_{2}=\left\{G \cup F: G \in \mathcal{I}_{1}, F \in \mathcal{I}_{2}\right\} .
$$

Proposition 2.1. [18] If $\mathcal{I}_{1}, \mathcal{I}_{2}$ are two ideals on a non-empty set $U$ and $A, B$ are two subsets of $U$. Then, the collection $\mathcal{I}_{1} \vee \mathcal{I}_{2}$ is satisfied the following conditions:

1. $\mathcal{I}_{1} \vee \mathcal{I}_{2} \neq \phi$,

2. $A \in \mathcal{I}_{1} \vee \mathcal{I}_{2}, B \subseteq A \Rightarrow B \in \mathcal{I}_{1} \vee \mathcal{I}_{2}$,

3. $A, B \in \mathcal{I}_{1} \vee \mathcal{I}_{2} \Rightarrow A \cup B \in \mathcal{I}_{1} \vee \mathcal{I}_{2}$.

It means that the collection $\mathcal{I}_{1} \vee \mathcal{I}_{2}$ is an ideal on $U$.

Definition 2.3. [8] Let $R$ be an arbitrary binary relation on a non-empty finite set $U$ and $x \in U$, then $<x>\breve{R}=\cup\{p R: x \in p R\} .<x>\breve{R}$ is the union of all right neighborhoods containing $x$.

Theorem 2.1. [8] Let $U$ be a universal set and $R_{1}, R_{2}$ be two similarity relations on $U$. If $R_{1} \subseteq R_{2}$, then $\left\langle x>\breve{R}_{1} \subseteq<x>\breve{R}_{2}, \forall x \in U\right.$.

Definition 2.4. [8] Let $R$ be a similarity relation on nonempty set $U$. For any subset $A \subseteq U$, the first kind of the lower and upper approximations, boundary regions, accuracy and roughness of $A$ according to $R$ is defined respectively by:

$\underline{\operatorname{apr}}_{R}(A)=\{x \in U:<x>\breve{R} \subseteq A\}$.

$\overline{\operatorname{apr}}_{R}(A)=\{x \in U:<x>\breve{R} \cap A \neq \phi\}$.

$\operatorname{Boundary}_{R}(A)=\overline{\operatorname{apr}}_{R}(A)-\underline{\operatorname{apr}}_{R}(A)$.

$\operatorname{Accuracy}_{R}(A)=\left|\frac{\operatorname{apr}_{R}(A)}{\overline{a p r}_{R}(A)}\right|, \overline{\operatorname{apr}}_{R}(A) \neq \phi$.

$\operatorname{Roughness}_{R}(A)=1-\operatorname{Accuracy}_{R}(A)$.

Definition 2.5. [8] Let $R$ be a similarity relation on nonempty set $U$. For any subset $A \subseteq U$, the second kind of the lower and upper approximations, boundary regions, accuracy and roughness of $A$ according to $R$ is defined respectively by:

$\underline{\underline{\operatorname{apr}^{\prime}}} R(A)=\cup\{<x>\breve{R}:<x>\breve{R} \subseteq A\}$.

${\overline{\overline{a p r^{\prime}}}}_{R}(A)=\left(\underline{a p r}_{R}^{\prime}\left(A^{c}\right)\right)^{c}$, where $A^{c}$ is the complement of $A$.

$\operatorname{Boundary}_{R}^{\prime}(A)=\overline{a p r}_{R}^{\prime}(A)-\underline{a p r}_{R}^{\prime}(A)$.

$\operatorname{Accuracy}_{R}^{\prime}(A)=\left|\underset{\underline{{\overline{a p r^{\prime}}}_{R}(A)}(A)}{\operatorname{apr}_{R}(A)}\right| \overline{\operatorname{apr}_{R}^{\prime}}(A) \neq \phi$.

$\operatorname{Roughness}_{R}^{\prime}(A)=1-\operatorname{Accuracy}_{R}^{\prime}(A)$. 
Definition 2.6. [8] Let $R$ be a similarity relation on nonempty set $U$. For any subset $A \subseteq U$, the third kind of the upper and lower approximations, boundary regions, accuracy and roughness of $A$ according to $R$ is defined respectively by:

$\overline{\operatorname{apr}^{\prime \prime}}{ }_{R}(A)=\cup\{<x>\breve{R}:<x>\breve{R} \cap A \neq \phi\}$.

$\underline{\operatorname{apr}}_{R}^{\prime \prime}(A)=\left(\overline{\operatorname{apr}}_{R}^{\prime \prime}\left(A^{c}\right)\right)^{c}$.

$\overline{B o u n d a r y}_{R}^{\prime \prime}(A)=\overline{\operatorname{apr}}_{R}^{\prime \prime}(A)-\underline{a p r}_{R}^{\prime \prime}(A)$.

$\operatorname{Accuracy}_{R}^{\prime \prime}(A)=\left|\underset{\underline{\operatorname{apr}_{R}^{\prime \prime}}}{R}(A)\right|, \mid \overline{\operatorname{apr}_{R}^{\prime \prime}}(A) \neq \phi$.

$\operatorname{Roughness}_{R}^{\prime \prime}(A)=1-\operatorname{Accuracy}_{R}^{\prime \prime}(A)$.

\section{The first method of generalized rough sets via ideals}

In this section, the first type of generalized rough approximations via ideals and the union of neighborhoods which generated by any binary relation is presented. The principle properties of these approximations is studied and compared to the previous ones in [8] and shown to be more general.

Definition 3.1. Let $R$ be a binary relation on a nonempty set $U$ and $\mathcal{I}$ be an ideal on $U$. For any subset $A \subseteq U$, the first kind of the generalized lower, upper approximations, boundary regions, accuracy and roughness of $A$ via ideal and according to $R$ is defined respectively by:

$\underline{\operatorname{apr}}_{R}^{\mathcal{I}}(A)=\left\{x \in U:<x>\breve{R} \cap A^{c} \in \mathcal{I}\right\}$.

$\overline{\operatorname{apr}}_{R}^{\mathcal{I}}(A)=\{x \in U:<x>\breve{R} \cap A \notin \mathcal{I}\}$.

$\operatorname{Boundary}_{R}^{\mathcal{I}}(A)=\overline{\operatorname{apr}}_{R}^{\mathcal{I}}(A)-\underline{a p r}_{R}^{\mathcal{I}}(A)$.

$\operatorname{Accuracy}_{R}^{\mathcal{I}}(A)=\frac{\left|\operatorname{apr}_{R}^{\mathcal{I}}(A)\right|}{\mid \overline{\operatorname{apr}_{R}^{\mathcal{I}}(A)}} \mid, \overline{\operatorname{apr}}_{R}^{\mathcal{I}}(A) \neq \phi$.

$\operatorname{Roughness}_{R}^{\mathcal{I}}(A)=1-\operatorname{Accuracy}_{R}^{\mathcal{I}}(A)$.

The properties of the current upper and lower approximations are studied in the following two propositions.

Proposition 3.1. Let $A, B \subseteq U, \mathcal{I}, \mathcal{J}$ be two ideals on $U$ and $R$ be a binary relation on $U$. Then, the following properties hold:

1. $\overline{\operatorname{apr}}_{R}^{\mathcal{I}}(\phi)=\phi$.

2. $A \subseteq B \Rightarrow \overline{a p r}_{R}^{\mathcal{I}}(A) \subseteq \overline{a p r}_{R}^{\mathcal{I}}(B)$.

3. $\overline{\operatorname{apr}}_{R}^{\mathcal{I}}(A \cap B) \subseteq \overline{a p r}_{R}^{\mathcal{I}}(A) \cap \overline{a p r}_{R}^{\mathcal{I}}(B)$.

4. $\overline{a p r}_{R}^{\mathcal{I}}(A \cup B)=\overline{a p r}_{R}^{\mathcal{I}}(A) \cup \overline{a p r}_{R}^{\mathcal{I}}(B)$.

5. $\overline{\operatorname{apr}}_{R}^{\mathcal{I}}(A)=\left(\underline{\operatorname{apr}}_{R}^{\mathcal{I}}\left(A^{c}\right)\right)^{c}$.

6. if $A \in \mathcal{I}$, then $\overline{\operatorname{apr}}_{R}^{\mathcal{I}}(A)=\phi$. 
7. if $\mathcal{I} \subseteq \mathcal{J}$, then $\overline{a p r}_{R}^{\mathcal{J}}(A) \subseteq \overline{a p r}_{R}^{\mathcal{I}}(A)$.

8. if $\mathcal{I}=P(U)$, then $\overline{\operatorname{apr}}{ }_{R}^{\mathcal{I}}(A)=\phi$.

9. $\overline{a p r}_{R}^{\mathcal{I} \cap \mathcal{J}}(A)=\overline{a p r}_{R}^{\mathcal{I}}(A) \cup \overline{a p r}_{R}^{\mathcal{J}}(A)$.

10. $\overline{a p r}_{R}^{\mathcal{I} \vee \mathcal{J}}(A)=\overline{a p r}_{R}^{\mathcal{I}}(A) \cap \overline{a p r}_{R}^{\mathcal{J}}(A)$.

\section{Proof.}

1.

$$
\begin{aligned}
\overline{\operatorname{apr}}_{R}^{\mathcal{I}}(\phi) & =\{x \in U:<x>\breve{R} \cap \phi \notin \mathcal{I}\} . \\
& =\phi .
\end{aligned}
$$

2. Let $x \in \overline{a p r}_{R}^{\mathcal{I}}(A)$. Then, $\langle x>\breve{R} \cap A \notin \mathcal{I}$. Since, $A \subseteq B$ and $\mathcal{I}$ is an ideal. Thus,

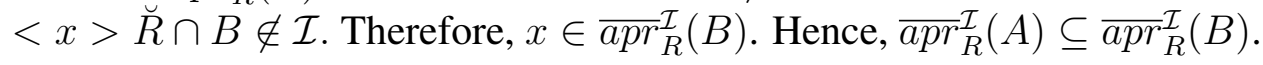

3. Immediately by part (2).

4. $\overline{a p r}_{R}^{\mathcal{I}}(A) \cup \overline{a p r}_{R}^{\mathcal{I}}(B) \subseteq \overline{a p r}_{R}^{\mathcal{I}}(A \cup B)$ by part (2). Let $x \in \overline{a p r}_{R}^{\mathcal{I}}(A \cup B)$. Then, $\langle x\rangle$ $\breve{R} \cap(A \cup B) \notin \mathcal{I}$. It follows that $((<x>\breve{R} \cap A) \cup(<x>R \cap \cap B)) \notin \mathcal{I}$. Therefore, $<x>\breve{R} \cap A \notin I$ or $<x>\breve{R} \cap B \notin \mathcal{I}$, that means $x \in \overline{a p r}_{R}^{\mathcal{I}}(A)$ or $x \in \overline{a p r}_{R}^{\mathcal{I}}(B)$. Then, $x \in \overline{a p r}_{R}^{\mathcal{I}}(A) \cup \overline{a p r}_{R}^{\mathcal{I}}(B)$. Thus, $\overline{a p r}_{R}^{\mathcal{I}}(A \cup B) \subseteq \overline{a p r}_{R}^{\mathcal{I}}(A) \cup \overline{a p r}_{R}^{\mathcal{I}}(B)$. Hence, $\overline{a p r}_{R}^{\mathcal{I}}(A \cup B)=$ $\overline{a p r}_{R}^{\mathcal{I}}(A) \cup \overline{a p r}_{R}^{\mathcal{I}}(B)$.

5.

$$
\begin{aligned}
\left(\underline{\operatorname{apr}}_{R}^{\mathcal{I}}\left(A^{c}\right)\right)^{c} & =(\{x \in U:<x>\breve{R} \cap A \in \mathcal{I}\})^{c} . \\
& =\{x \in U:<x>\breve{R} \cap A \notin \mathcal{I}\} . \\
& =\overline{a p r}_{R}^{\mathcal{I}}(A) .
\end{aligned}
$$

\section{Straightforward by Definition 3.1.}

7. Let $x \in \overline{a p r}_{R}^{\mathcal{J}}(A)$. Then, $\langle x>\breve{R} \cap A \notin \mathcal{J}$. Since, $\mathcal{I} \subseteq \mathcal{J}$. Thus, $\langle x>\breve{R} \cap A \notin \mathcal{I}$. Therefore, $x \in \overline{a p r}_{R}^{\mathcal{I}}(A)$. Hence, $\overline{a p r}{ }_{R}^{\mathcal{J}}(A) \subseteq \overline{a p r}_{R}^{\mathcal{I}}(A)$.

\section{Straightforward by Definition 3.1.}

9.

$$
\begin{aligned}
\overline{a p r}_{R}^{\mathcal{I} \cap \mathcal{J}}(A) & =\{x \in U:<x>\breve{R} \cap A \notin \mathcal{I} \cap \mathcal{J}\} . \\
& =\{x \in U:<x>\breve{R} \cap A \notin \mathcal{I}\} \text { or }\{x \in U:<x>\breve{R} \cap A \notin \mathcal{J}\} . \\
& =\{x \in U:<x>\breve{R} \cap A \notin \mathcal{I}\} \cup\{x \in U:<x>\breve{R} \cap A \notin \mathcal{J}\} . \\
& =\overline{\operatorname{apr}}_{R}^{\mathcal{I}}(A) \cup \overline{\operatorname{apr}}_{R}^{\mathcal{J}}(A) .
\end{aligned}
$$


10.

$$
\begin{aligned}
\overline{a p r}_{R}^{\mathcal{I} \vee \mathcal{J}}(A) & =\{x \in U:<x>\breve{R} \cap A \notin \mathcal{I} \vee \mathcal{J}\} \\
& =\{x \in U:<x>\breve{R} \cap A \notin \mathcal{I} \cup \mathcal{J}\} . \\
& =\{x \in U:<x>\breve{R} \cap A \notin \mathcal{I}\} \text { and }\{x \in U:<x>\breve{R} \cap A \notin \mathcal{J}\} \\
& =\{x \in U:<x>\breve{R} \cap A \notin \mathcal{I}\} \cap\{x \in U:<x>\breve{R} \cap A \notin \mathcal{J}\} . \\
& =\overline{a p r}_{R}^{\mathcal{I}}(A) \cap \overline{a p r}_{R}^{\mathcal{J}}(A) .
\end{aligned}
$$

Proposition 3.2. Let $A, B \subseteq U, \mathcal{I}, \mathcal{J}$ be two ideals on $U$ and $R$ be a binary relation on $U$. Then, the following properties hold:

1. $\operatorname{apr}_{R}^{\mathcal{I}}(U)=U$.

2. $A \subseteq B \Rightarrow \underline{a p r}_{R}^{\mathcal{I}}(A) \subseteq \underline{a p r}_{R}^{\mathcal{I}}(B)$.

3. $\operatorname{apr}_{R}^{\mathcal{I}}(A) \cup \underline{a p r}_{R}^{\mathcal{I}}(B) \subseteq \underline{a p r}_{R}^{\mathcal{I}}(A \cup B)$.

4. $\underline{\operatorname{apr}}_{R}^{\mathcal{I}}(A \cap B)=\underline{a p r}_{R}^{\mathcal{I}}(A) \cap \underline{\operatorname{apr}}_{R}^{\mathcal{I}}(B)$.

5. ${ }_{\operatorname{apr}_{R}^{\mathcal{I}}}(A)=\left(\overline{\operatorname{apr}}_{R}^{\mathcal{I}}\left(A^{c}\right)\right)^{c}$.

6. if $A^{c} \in \mathcal{I}$, then $\underline{\operatorname{apr}}_{R}^{\mathcal{I}}(A)=U$.

7. if $\mathcal{I} \subseteq \mathcal{J}$, then $\underline{\operatorname{apr}}_{R}^{\mathcal{I}}(A) \subseteq \underline{a p r}_{R}^{\mathcal{J}}(A)$.

8. if $\mathcal{I}=P(U)$, then $\underline{\operatorname{apr}}_{R}^{\mathcal{I}}(A)=U$.

9. $\underline{a p r}_{R}^{\mathcal{I} \cap \mathcal{J}}(A)=\underline{a p r}_{R}^{\mathcal{I}}(A) \cap \underline{a p r}_{R}^{\mathcal{J}}(A)$.

\section{Proof.}

1.

$$
\begin{aligned}
\operatorname{apr}_{R}^{\mathcal{I}}(U) & =\{x \in U:<x>\breve{R} \cap \phi \in \mathcal{I}\} \\
& =U
\end{aligned}
$$

2. Let $x \in \underline{a p r}_{R}^{\mathcal{I}}(A)$. Then, $\left\langle x>\breve{R} \cap A^{c} \in \mathcal{I}\right.$. Since, $B^{c} \subseteq A^{c}$ and $\mathcal{I}$ is an ideal. Thus, $<x>\breve{R} \cap B^{c} \in \mathcal{I}$. Therefore, $x \in \underline{a p r}_{R}^{\mathcal{I}}(B)$. Hence, $\underline{a p r}_{R}^{\mathcal{I}}(A) \subseteq \underline{a p r_{R}^{\mathcal{I}}}(B)$.

3. Immediately by part (2).

4. $\underline{a p r}_{R}^{\mathcal{I}}(A) \cap \underline{a p r}_{R}^{\mathcal{I}}(B) \supseteq \underline{a p r}_{R}^{\mathcal{I}}(A \cap B)$ by part (2). Let $x \in \underline{a p r}_{R}^{\mathcal{I}}(A) \cap \underline{a p r}_{R}^{\mathcal{I}}(B)$. Then, $<x>\breve{R} \cap \overline{A^{c}} \in \mathcal{I}$ and $<x>\breve{R} \cap B^{c} \in \mathcal{I}$. It follows that $\left(<x>\breve{R} \cap\left(A^{c} \cup B^{c}\right)\right) \in \mathcal{I}$. So, $\left(<x>\breve{R} \cap(A \cap B)^{c}\right) \in \mathcal{I}$. Therefore, $x \in \underline{a p r}_{R}^{\mathcal{I}}(A \cap B)$. Thus, $\underline{a p r_{R}^{\mathcal{I}}}(A) \cap \underline{a p r}_{R}^{\mathcal{I}}(B) \subseteq$ $\underline{a p r}_{R}^{\mathcal{I}}(A \cap B)$. Hence, $\underline{a p r_{R}^{\mathcal{I}}}(A) \cap \underline{a p r_{R}^{\mathcal{I}}}(B)=\underline{a p r}_{R}^{\mathcal{I}}(A \cap B)$. 
5.

$$
\begin{aligned}
\left(\overline{\operatorname{apr}}_{R}^{\mathcal{I}}\left(A^{c}\right)\right)^{c} & =\left(\left\{x \in U:<x>\breve{R} \cap A^{c} \notin \mathcal{I}\right\}\right)^{c} . \\
& =\left\{x \in U:<x>\breve{R} \cap A^{c} \in \mathcal{I}\right\} . \\
& =\underline{\operatorname{apr}}_{R}^{\mathcal{I}}(A) .
\end{aligned}
$$

6. Straightforward by Definition 3.1.

7. Let $x \in \underline{a p r}_{R}^{\mathcal{I}}(A)$. Then, $<x>\breve{R} \cap A^{c} \in \mathcal{I}$. Since, $\mathcal{I} \subseteq \mathcal{J}$. Thus, $<x>\breve{R} \cap A^{c} \in \mathcal{J}$. Therefore, $x \in \underline{a p r}_{R}^{\mathcal{I}}(A)$. Hence, $\underline{a p r}_{R}^{\mathcal{I}}(A) \subseteq \underline{a p r}_{R}^{\mathcal{J}}(A)$.

8. Straightforward by Definition 3.1.

9.

$$
\begin{aligned}
\underline{\operatorname{apr}}_{R}^{\mathcal{I} \cap \mathcal{J}}(A) & =\left\{x \in U:<x>\breve{R} \cap A^{c} \in \mathcal{I} \cap \mathcal{J}\right\} . \\
& =\left\{x \in U:<x>\breve{R} \cap A^{c} \in \mathcal{I}\right\} \text { and }\left\{x \in U:<x>\breve{R} \cap A^{c} \in \mathcal{J}\right\} . \\
& =\left\{x \in U:<x>\breve{R} \cap A^{c} \in \mathcal{I}\right\} \cap\left\{x \in U:<x>\breve{R} \cap A^{c} \in \mathcal{J}\right\} . \\
& =\underline{a p r}_{R}^{\mathcal{I}}(A) \cap \underline{\operatorname{apr}}_{R}^{\mathcal{J}}(A) .
\end{aligned}
$$

Remark 3.1. The following examples show that

1. the converse of parts 2, 6, 7 and 8 in Propositions 3.1 and 3.2 is not necessarily to be true in general.

2. the inclusion of part 3 in Propositions 3.1 and 3.2 can not be replaced by equality relation in general.

Example 3.1. (i) Let $U=\{a, b, c, d\}, \mathcal{I}=\{\phi,\{a\},\{b\},\{c\},\{a, b\},\{a, c\},\{b, c\},\{a, b, c\}\}$ and $R=\{(a, b),(a, c),(b, c),(b, d),(c, a),(c, d)\}$ be a binary relation defined on $U$ thus $\langle a\rangle$ $\breve{R}=\{a, d\},<b>\breve{R}=\{b, c\},<c>\breve{R}=\{b, c, d\},<d>\breve{R}=\{a, c, d\}$. For part 2, take

(a) $A=\{a\}, B=\{d\}$, then $\overline{a p r}_{R}^{\mathcal{I}}(A)=\phi, \overline{a p r}_{R}^{\mathcal{I}}(B)=\{a, c, d\}$. Therefore, $\overline{a p r}_{R}^{\mathcal{I}}(A) \subseteq$ $\overline{\operatorname{apr}}_{R}^{\mathcal{I}}(B)$, but $A \nsubseteq B$.

(b) $A=\{b\}, B=\{a, c, d\}$, then $\underline{a p r}_{R}^{\mathcal{I}}(A)=\{b\}, \underline{a p r_{R}^{\mathcal{I}}}(B)=U$. Therefore, apr $\underline{R}_{R}^{\mathcal{I}}(A) \subseteq$ $\operatorname{apr}_{R}^{\mathcal{I}}(B)$, but $A \nsubseteq B$.

(ii) Let $U=\{a, b, c, d\}, \mathcal{J}=\{\phi,\{a\}\}, \mathcal{I}=\{\phi,\{d\}\}$ and $R=\{(a, a),(b, b),(c, c)\}$ be a binary relation defined on $U$ thus $<a>\breve{R}=\{a\},<b>\breve{R}=\{b\},<c>\breve{R}=\{c\},<d>\breve{R}=\phi$.

1. for part 6 , take

(a) $A=\{a, d\}$, then $\overline{a p r}_{R}^{\mathcal{J}}(A)=\phi$. Therefore, $\overline{\operatorname{apr}}_{R}^{\mathcal{J}}(A)=\phi$, but $A \notin \mathcal{J}$.

(b) $A=\{b, c\}$, then $\underline{\operatorname{apr}}_{R}^{\mathcal{J}}(A)=U$. Therefore, $\underline{\operatorname{apr}}_{R}^{\mathcal{J}}(A)=U$, but $A^{c} \notin \mathcal{J}$. 
2. for part 7 , take

(a) $A=\{a, d\}$, then $\overline{a p r}_{R}^{\mathcal{I}}(A)=\{a\}, \overline{a p r}_{R}^{\mathcal{J}}(A)=\phi$. Therefore, $\overline{a p r}_{R}^{\mathcal{J}}(A) \subseteq \overline{a p r}_{R}^{\mathcal{I}}(A)$, but $\mathcal{I} \nsubseteq \mathcal{J}$.

(b) $A=\{b, c\}$, then $\frac{a p r}{\mathcal{I}}_{R}^{\mathcal{I}}(A)=\{b, c, d\}, \underline{a p r}_{R}^{\mathcal{J}}(A)=U$. Therefore, $\underline{a p r}_{R}^{\mathcal{I}}(A) \subseteq$ $\operatorname{apr}_{R}^{\mathcal{J}}(A)$, but $\mathcal{I} \nsubseteq \overline{\mathcal{J}}$.

3. for part 8 , take

(a) $A=\{a, d\}$, then $\overline{a p r}_{R}^{\mathcal{J}}(A)=\phi$, but $\mathcal{J} \neq P(U)$.

(b) $A=\{b, c\}$, then $\underline{\operatorname{apr}}_{R}^{\mathcal{J}}(A)=U$, but $\mathcal{J} \neq P(U)$.

(iii) Let $U=\{a, b, c, d\}, \mathcal{I}=\{\phi,\{d\}\}$ and $R=\Delta \cup\{(b, a),(c, a),(d, a)\}$ be a binary relation defined on $U$, (where $\Delta$ is the identity relation and equal to $\{(a, a),(b, b),(c, c),(d, d)\})$ thus $<a>\breve{R}=U,<b>\breve{R}=\{a, b\},<c>\breve{R}=\{a, c\},<d>\breve{R}=\{a, d\}$. For part 3, take

(a) $A=\{a, d\}, B=\{b, c\}, A \cap B=\phi$, then $\overline{a p r}_{R}^{\mathcal{I}}(A)=U, \overline{a p r}_{R}^{\mathcal{I}}(B)=\{a, b, c\}, \overline{a p r}_{R}^{\mathcal{I}}($

$A \cap B)=\phi$. Therefore, $\overline{a p r}_{R}^{\mathcal{I}}(A) \cap \overline{a p r}_{R}^{\mathcal{I}}(B)=\{a, b, c\} \neq \phi=\overline{a p r}_{R}^{\mathcal{I}}(A \cap B)$.

(b) $A=\{a, d\}, B=\{b, c\}, A \cup B=U$, then $\underset{a p r_{R}^{\mathcal{I}}}{ }(A)=\{d\}, \underline{a p r}_{R}^{\mathcal{I}}(B)=\phi, \overline{a p r}_{R}^{\mathcal{I}}(A \cup$ $B)=U$. Therefore, $\left.\left.\underline{a p r}_{R}^{\mathcal{I}}(A) \cup \underline{a p r}_{R}^{\mathcal{I}}(B)=\overline{\{d}\right\} \neq U=\underline{a p r}_{R}^{\overline{\mathcal{I}}(A} \cup B\right)$.

Remark 3.2. There are some properties which are not hold or satisfied in the first type.

(i) In Example 3.1 (i) take

1. $A=\{a\}$, then $\overline{a p r}_{R}^{\mathcal{I}}(A)=\phi$. Hence, $A \nsubseteq \overline{a p r}_{R}^{\mathcal{I}}(A)$.

2. $A=\{b, c, d\}$, then $\underline{a p r}_{R}^{\mathcal{I}}(A)=U$. Hence, $\underline{a p r}_{R}^{\mathcal{I}}(A) \nsubseteq A$.

3. $A=U$, then $\overline{a p r}_{R}^{\mathcal{I}}(U)=\{a, c, d\}$. Hence, $\overline{a p r}_{R}^{\mathcal{I}}(U) \neq U$.

4. $A=\phi$, then $\underline{a p r}_{R}^{\mathcal{I}}(\phi)=\{b\}$. Hence, $\underline{a p r}_{R}^{\mathcal{I}}(\phi) \neq \phi$.

(ii) In Example 3.1 (iii) take

1. $A=\{b, c\}$, then $\overline{a p r}_{R}^{\mathcal{I}}(A)=\{a, b, c\}, \overline{a p r}_{R}^{\mathcal{I}}\left(\overline{a p r}_{R}^{\mathcal{I}}(A)\right)=U$. Hence, $\overline{a p r}_{R}^{\mathcal{I}}(A) \neq$ $\overline{\operatorname{apr}}_{R}^{\mathcal{I}}\left(\overline{a p r}_{R}^{\mathcal{I}}(A)\right)$.

2. $A=\{a, d\}$, then $\underline{a p r_{R}^{\mathcal{I}}}(A)=\{d\}, \underline{a p r_{R}^{\mathcal{I}}}\left(a p r_{R}^{\mathcal{I}}(A)\right)=\phi$. Hence, $\underline{a p r}{ }_{R}^{\mathcal{I}}(A) \neq \underline{a p r_{R}^{\mathcal{I}}}$ $\left.\underline{\operatorname{apr}}_{R}^{\mathcal{I}}(A)\right)$.

(iii)

Example 3.2. Let $U=\{a, b, c, d\}, \mathcal{I}=\{\phi,\{a\}\}$ and $R=\Delta \cup\{(a, b),(b, a),(c, a),(c, b),(d, a),(d, b)\}$ be a binary relation defined on $U$ thus $<a>R=<b>R=U,<c>R=\{a, b, c\},<$ $d>\breve{R}=\{a, b, d\}$. It is clear that, if

1. $A=\{c\}$, then $\overline{a p r}_{R}^{\mathcal{I}}(A)=\{a, b, c\}, \underline{a p r}_{R}^{\mathcal{I}}\left(\overline{a p r}_{R}^{\mathcal{I}}(A)\right)=\{c\}$. Hence, $\overline{a p r}_{R}^{\mathcal{I}}(A) \nsubseteq$ $\underline{a p r}_{R}^{\mathcal{I}}\left(\overline{a p r}_{R}^{\mathcal{I}}(A)\right)$.

2. $A=\{a, b, d\}$, then $\underline{a p r}_{R}^{\mathcal{I}}(A)=\{d\}, \overline{a p r}_{R}^{\mathcal{I}}\left(\underline{a p r}_{R}^{\mathcal{I}}(A)\right)=\{a, b, d\}$. Hence, $\overline{a p r}{ }_{R}^{\mathcal{I}}\left(a p r_{R}^{\mathcal{I}}\right.$ $(A)) \nsubseteq \underline{a p r}_{R}^{\mathcal{I}}(A)$. 
The following theorem shows that the boundary of a subset decreases and the accuracy increases as the ideal on $U$ increases.

Theorem 3.1. Let $A \subseteq U, \mathcal{I}, \mathcal{J}$ be two ideals on $U$ and $R$ be a binary relation on $U$. If $\mathcal{I} \subseteq \mathcal{J}$. Then,

1. $\operatorname{Boundary}_{R}^{\mathcal{J}}(A) \subseteq \operatorname{Boundary}_{R}^{\mathcal{I}}(A)$.

2. $\operatorname{Accuracy}_{R}^{\mathcal{I}}(A) \leq \operatorname{Accuracy}_{R}^{\mathcal{J}}(A)$.

\section{Proof.}

1. Let $x \in$ Boundary $_{R}^{\mathcal{J}}(A)$. Then, $x \in \overline{a p r}_{R}^{\mathcal{J}}(A)-\operatorname{apr}_{R}^{\mathcal{J}}(A)$. So, $x \in \overline{a p r}_{R}^{\mathcal{J}}(A)$ and $x \in$ $\left(\operatorname{apr}_{R}^{\mathcal{J}}(A)\right)^{c}$. Hence, $x \in \overline{a p r}_{R}^{\mathcal{I}}(A)$ and $x \in\left(\underline{a p r}_{R}^{\mathcal{I}}\left(\overline{A))^{c}}\right.\right.$ by Propositions 3.1 and 3.2 part 7 . It follows that $x \in \operatorname{Boundar}_{R}^{\mathcal{I}}(A)$. Therefore, $\operatorname{Boundar}_{R}^{\mathcal{J}}(A) \subseteq \operatorname{Boundary}_{R}^{\mathcal{I}}(A)$.

2.

$$
\begin{aligned}
\operatorname{Accuracy}_{R}^{\mathcal{I}}(A) & =\left|\frac{\overline{a p r}_{R}^{\mathcal{I}}(A)}{\overline{\operatorname{apr}}_{R}^{\mathcal{I}}(A)}\right| . \\
& \leq\left|\frac{\overline{a p r}_{R}^{\mathcal{J}}(A)}{\overline{\operatorname{apr}_{R}^{\mathcal{J}}(A)}}\right| . \\
& =\operatorname{Accuracy}_{R}^{\mathcal{J}}(A) .
\end{aligned}
$$

Remark 3.3. Example 3.1 (ii) shows that the converse of parts 1 and 2 in Theorem 3.1 is not necessary to be true in general. Take, $A=\{b, c\}$, then

1. $\operatorname{Boundary}_{R}^{\mathcal{J}}(A)=\phi \subseteq \phi=\operatorname{Boundary}_{R}^{\mathcal{I}}(A)$, but $\mathcal{I} \nsubseteq \mathcal{J}$.

2. $\operatorname{Accuracy}_{R}^{\mathcal{I}}(A)=\frac{3}{2}<2=\operatorname{Accuracy}_{R}^{\mathcal{J}}(A)$, but $\mathcal{I} \nsubseteq \mathcal{J}$.

The following theorem shows that the corresponding upper and lower approximations, boundary regions and accuracy measures of the first type of approximations are monotonic.

Theorem 3.2. Let $A \subseteq U, \mathcal{I}$ be an ideal on $U$ and $R_{1}, R_{2}$ be two similarity relations on $U$. If $R_{1} \subseteq R_{2}$, then

1. $\overline{\operatorname{apr}}_{R_{1}}^{\mathcal{I}}(A) \subseteq \overline{\operatorname{apr}}_{R_{2}}^{\mathcal{I}}(A)$.

2. $\operatorname{apr}_{R_{2}}^{\mathcal{I}}(A) \subseteq \underline{a p r^{\mathcal{I}}}(A)$.

3. Boundary $\mathcal{R}_{1}^{\mathcal{I}}(A) \subseteq \operatorname{Boundar} y_{R_{2}}^{\mathcal{I}}(A)$.

4. $\operatorname{Accuracy}_{R_{2}}^{\mathcal{I}}(A) \leq \operatorname{Accuracy}_{R_{1}}^{\mathcal{I}}(A)$.

\section{Proof.}


1. Let $x \in \overline{a p r}_{R_{1}}^{\mathcal{I}}(A)$. Then, $\left\langle x>\breve{R_{1}} \cap A \notin \mathcal{I}\right.$. Since, $<x>\breve{R_{1}} \subseteq<x>\breve{R_{2}}$ (by Theorem 2.1 [8]). It follows that $<x>\breve{R_{2}} \cap A \notin \mathcal{I}$. Thus, $x \in \overline{a p r}_{R_{2}}^{\mathcal{I}}(A)$. Hence, $\overline{a p r} \bar{R}_{R_{1}}^{\mathcal{I}}(A) \subseteq \overline{a p r}_{R_{2}}^{\mathcal{I}}(A)$.

2. Let $x \in \operatorname{apr}_{R_{2}}^{\mathcal{I}}(A)$. Then, $\left\langle x>\breve{R}_{2} \cap A^{c} \in \mathcal{I}\right.$. Since, $\left\langle x>\breve{R}_{1} \subseteq<x>\breve{R}_{2}\right.$ (by Theorem 2.1 [8]). It follows that $\left\langle x>\breve{R_{1}} \cap A^{c} \in \mathcal{I}\right.$. Thus, $x \in \underline{a p r}_{R_{1}}^{\mathcal{I}}(A)$. Hence, $\underline{\operatorname{apr}}_{R_{2}}^{\mathcal{I}}(A) \subseteq \underline{\operatorname{apr}}_{R_{1}}^{\mathcal{I}}(A)$.

3. Let $x \in$ Boundary $_{R_{1}}^{\mathcal{I}}(A)$. Then, $x \in \overline{a p r} r_{R_{1}}^{\mathcal{I}}(A)-\operatorname{apr}_{R_{1}}^{\mathcal{I}}(A)$. So, $x \in \overline{\operatorname{apr}_{R_{1}}^{\mathcal{I}}}(A)$ and $x \in$ $\left(\underline{a p r}_{R_{1}}^{\mathcal{I}}(A)\right)^{c}$. Thus, $x \in \overline{a p r} r_{R_{2}}^{\mathcal{I}}(A)$ and $x \in\left(\underline{a p r}_{R_{2}}^{\mathcal{I}}(A)\right)^{c}$ by parts 1 and 2. Hence, $x \in$ $\operatorname{Boundary}_{R_{2}}^{\mathcal{I}}(A)$. Therefore, Boundary ${ }_{R_{1}}^{\mathcal{I}}(A) \subseteq$ Boundary $_{R_{2}}^{\mathcal{I}}(A)$.

4.

$$
\begin{aligned}
\operatorname{Accuracy}_{R_{2}}^{\mathcal{I}}(A) & =\mid \frac{\operatorname{apr}_{R_{2}}^{\mathcal{I}}(A)}{\overline{\overline{a p r} r_{R_{2}}^{\mathcal{I}}(A)} \mid .} \\
& \leq\left|\frac{\operatorname{apr}_{R_{1}}^{\mathcal{I}}(A)}{\overline{\overline{a p r}} R_{R_{1}}^{\mathcal{I}}(A)}\right| . \\
& =\operatorname{Accuracy}_{R_{1}}^{\mathcal{I}}(A) .
\end{aligned}
$$

The following example shows that the inclusion and the less than in Theorem 3.2 can not be replaced by equality relation in general.

Example 3.3. Let $U=\{a, b, c, d\}, \mathcal{I}=\{\phi,\{b\},\{c\},\{d\},\{b, c\},\{b, d\},\{c, d\},\{b, c, d\}\}$ and $R_{1}=\Delta \cup\{(a, b),(b, a)\}, R_{2}=\Delta \cup\{(a, b),(b, a),(c, a),(a, c)\}$ be two similarity relation defined on $U$ thus $\left\langle a>\breve{R}_{1}=\left\langle b>\breve{R}_{1}=\{a, b\},<c>\breve{R}_{1}=\{c\},<d>\breve{R}_{1}=\{d\},<a>\right.\right.$ $\breve{R}_{2}=<b>\breve{R}_{2}=<c>\breve{R}_{2}=\{a, b, c\},<d>\breve{R}_{2}=\{d\}$. Take

(i) $A=\{a, d\}$, then

$$
\begin{aligned}
& \text { 1. } \overline{\operatorname{apr}}_{R_{1}}^{\mathcal{I}}(A)=\{a, b\} \neq\{a, b, c\}=\overline{a p r}_{R_{2}}^{\mathcal{I}}(A) . \\
& \text { 2. } \operatorname{Accuracy}_{R_{1}}^{\mathcal{I}}(A)=2 \neq \frac{4}{3}=\operatorname{Accuracy}_{R_{2}}^{\mathcal{I}}(A) .
\end{aligned}
$$

(ii) $A=\{b, c\}$, then $\underline{a p r}_{R_{1}}^{\mathcal{I}}(A)=\{c, d\} \neq\{d\}=\underline{a p r}_{R_{2}}^{\mathcal{I}}(A)$.

The following theorem presents the relationships between the current approximations in Definition 3.1 and the previous one in Definition 2.4 [8]. It shows that the present method in Definition 3.1 reduces the boundary region by increasing the lower approximations and decreasing the upper approximations with the comparison of Dai et al.'s method in Definition 2.4 [8]. Additionally, it shows that the current accuracy in Definition 3.1 is greater than the previous one in Definition 2.4 [8].

Theorem 3.3. Let $A \subseteq U, \mathcal{I}$ be an ideal on $U$ and $R$ be a similarity relation on a nonempty set $U$. Then, 
1. $\overline{\operatorname{apr}}_{R}^{\mathcal{I}}(A) \subseteq \overline{a p r}_{R}(A)$.

2. $\underline{\operatorname{apr}}_{R}(A) \subseteq \underline{\operatorname{apr}}_{R}^{\mathcal{I}}(A)$.

3. Boundary $_{R}^{\mathcal{I}}(A) \subseteq$ Boundary $_{R}(A)$.

4. $\operatorname{Accuracy}_{R}(A) \leq \operatorname{Accuracy}_{R}^{\mathbb{I}}(A)$.

\section{Proof.}

(1) Let $x \in \overline{a p r}_{R}^{\mathcal{I}}(A)$. Then, $\langle x>\breve{R} \cap A \notin \mathcal{I}$. Hence, $<x>\breve{R} \cap A \neq \phi$. Therefore, $x \in \overline{a p r}_{R}(A)$. So, $\overline{a p r}_{R}^{\mathcal{I}}(A) \subseteq \overline{a p r}_{R}(A)$.

(2) Let $x \in \underline{a p r}_{R}(A)$. Then, $<x>\breve{R} \subseteq A$. Hence, $<x>\breve{R} \cap A^{c} \in \mathcal{I}$. Therefore, $x \in \underline{a p r}_{R}^{\mathcal{I}}(A)$. So, $\underline{a p r}_{R}(A) \subseteq \underline{a p r}_{R}^{\mathcal{I}}(A)$.

(3) and (4) Immediately by parts (1) and 2.

Remark 3.4. Example 3.3 shows that the inclusion and the less than in Theorem 3.3 can not be replaced by equality relation in general. Take $A=\{a, d\}$, then

1. $\overline{\operatorname{apr}}_{R_{1}}^{\mathcal{I}}(A)=\{a, b\} \neq\{a, b, d\}=\overline{a p r}_{R_{1}}(A)$.

2. $\underline{a p r}_{R_{1}}^{\mathcal{I}}(A)=U \neq\{d\}=\underline{a p r}_{R_{1}}(A)$.

3. Boundary $y_{R_{1}}^{\mathcal{I}}(A)=\phi \neq\{a, b\}=$ Boundary $_{R_{1}}(A)$.

4. $\operatorname{Accuracy}_{R_{1}}^{\mathcal{I}}(A)=2 \neq \frac{1}{3}=\operatorname{Accuracy}_{R_{1}}(A)$.

\section{The second method of generalized rough sets via ideals}

The aim of this section is to propose the second type of extension rough approximations by utilizing the notion of ideals and the union of neighborhoods which determined by any binary relation. The characteristics of these approximations are suggested. Moreover, the relationships between these approximations and the first type of approximations in the previous section are presented. Comparisons between these approximations and the ones in [8] are introduced.

Definition 4.1. Let $R$ be a binary relation on a nonempty set $U$ and $\mathcal{I}$ be an ideal on $U$. For any subset $A \subseteq U$, the second kind of the generalized lower, upper approximations, boundary regions, accuracy and roughness of $A$ via ideal and according to $R$ is defined respectively by:

$\underline{\underline{\operatorname{apr}_{R}^{\mathcal{I}}}}(A)=\left\{x \in A:<x>\breve{R} \cap A^{c} \in \mathcal{I}\right\}$.

$\overline{\overline{\operatorname{apr}}}_{R}^{\mathcal{I}}(A)=A \cup \overline{\operatorname{apr}}_{R}^{\mathcal{I}}(A)$.

$\underline{B o u n d a r y}_{R}^{\mathcal{I}}(A)=\overline{\overline{a p r}}_{R}(A)-\underline{\underline{\operatorname{apr}^{\mathcal{I}}}}(A)$.

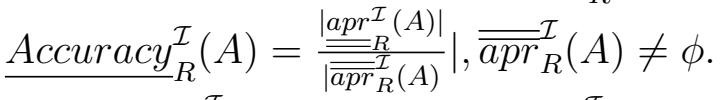

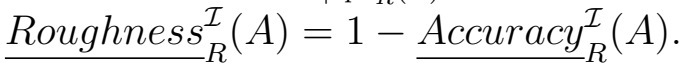


Proposition 4.1. Let $A \subseteq U, \mathcal{I}, \mathcal{J}$ be two ideals on $U$ and $R$ be a binary relation on $U$. Then, the following properties hold:

1. $A \subseteq \overline{\overline{a p r}}_{R}^{\mathcal{I}}(A)$ equality hold if $A=\phi$ or $U$.

2. $A \subseteq B \Rightarrow \overline{\overline{a p r}}_{R}^{\mathcal{I}}(A) \subseteq \overline{\overline{a p r}}_{R}^{\mathcal{I}}(B)$.

3. $\overline{\overline{a p r}}_{R}^{\mathcal{I}}(A) \subseteq \overline{\overline{a p r}}_{R}^{\mathcal{I}}\left(\overline{\overline{a p r}}_{R}^{\mathcal{I}}(A)\right)$.

4. $\overline{\overline{a p r}}_{R}^{\mathcal{I}}(A \cap B) \subseteq \overline{\overline{a p r}}_{R}^{\mathcal{I}}(A) \cap \overline{\overline{a p r}}_{R}^{\mathcal{I}}(B)$.

5. $\overline{\overline{a p r}}_{R}^{\mathcal{I}}(A \cup B)=\overline{\overline{a p r}}_{R}^{\mathcal{I}}(A) \cup \overline{\overline{a p r}}_{R}^{\mathcal{I}}(B)$.

6. $\overline{\overline{a p r}}_{R}^{\mathcal{I}}(A)=\left(\underline{\underline{a p r}}_{R}^{\mathcal{I}}\left(A^{c}\right)\right)^{c}$.

7. if $A \in \mathcal{I}$, then $\overline{\overline{a p r}}_{R}^{\mathcal{I}}(A)=A$.

8. if $\mathcal{I} \subseteq \mathcal{J}$, then $\overline{\overline{a p r}}_{R}^{\mathcal{J}}(A) \subseteq \overline{\overline{a p r}}_{R}^{\mathcal{I}}(A)$.

9. if $\mathcal{I}=P(U)$, then $\overline{\overline{a p r}}_{R}^{\mathcal{I}}(A)=A$.

10. $\overline{\overline{a p r}}_{R}^{\mathcal{I} \cap \mathcal{J}}(A)=\overline{\overline{a p r}}_{R}^{\mathcal{I}}(A) \cup \overline{\overline{a p r}}_{R}^{\mathcal{J}}(A)$.

11. $\overline{\overline{a p r}}_{R}^{\mathcal{I} \vee \mathcal{J}}(A)=\overline{\overline{a p r}}_{R}^{\mathcal{I}}(A) \cap \overline{\overline{a p r}}_{R}^{\mathcal{J}}(A)$.

Proof. Similar to Proposition 3.1.

Proposition 4.2. Let $A, B \subseteq U, \mathcal{I}, \mathcal{J}$ be two ideals on $U$ and $R$ be a binary relation on $U$. Then, the following properties hold:

1. $\underline{\operatorname{apr}}_{R}^{\mathcal{I}}(A) \subseteq$ A equality hold if $A=\phi$ or $U$.

2. $A \subseteq B \Rightarrow \underline{\underline{\operatorname{apr}^{\mathcal{I}}}}(A) \subseteq \underline{\underline{\operatorname{apr}^{\mathcal{I}}}} R(B)$.

3. $\left.\underset{\underline{\operatorname{apr}^{\mathcal{I}}}}{=} \stackrel{\underline{a p r}^{\mathcal{I}}}{=}(A)\right) \subseteq \underline{\underline{a p r}}_{R}^{\mathcal{I}}(A)$.

4. $\underline{\underline{a p r}}_{R}^{\mathcal{I}}(A) \cup \underline{\underline{a p r}}_{R}^{\mathcal{I}}(B) \subseteq \underline{\underline{a p r}}_{R}^{\mathcal{I}}(A \cup B)$.

5. $\underset{\underline{\operatorname{apr}^{\mathcal{I}}}}{R}(A \cap B)=\underline{\underline{a p r}}_{R}^{\mathcal{I}}(A) \cap \underline{\underline{a p r}}_{R}^{\mathcal{I}}(B)$.

6. $\underline{\underline{a p r}}_{R}^{\mathcal{I}}(A)=\left(\underline{\underline{a p r}}_{R}^{\mathcal{I}}\left(A^{c}\right)\right)^{c}$.

7. if $A^{c} \in \mathcal{I}$, then $\underline{\underline{\operatorname{apr}^{\mathcal{I}}}} R$

8. if $\mathcal{I} \subseteq \mathcal{J}$, then $\underline{\underline{a p r}}_{R}^{\mathcal{I}}(A) \subseteq \underline{\underline{a p r}}_{R}^{\mathcal{J}}(A)$. 
9. if $\mathcal{I}=P(U)$, then $\underset{\underline{a p r}}{\underline{I}}(A)=A$.

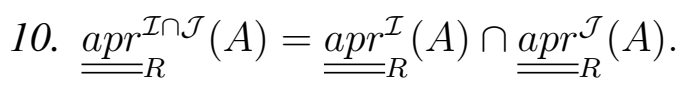

Proof. Similar to Proposition 3.2.

Remark 4.1. (i) Example 3.1 (i) also shows that the converse of parts 2, 7 and 9 in Propositions 4.1 and 4.2 is not necessarily to be true in general.

(a) For part 2, take

1. $A=\{a\}, B=\{d\}$, then $\overline{\overline{a p r}}_{R}^{\mathcal{I}}(A)=\{a\} \subseteq\{a, c, d\}=\overline{\overline{a p r}}_{R}^{\mathcal{I}}(B)$, but $A \nsubseteq B$.

2. $A=\{a, b, c\}, B=\{b, c, d\}$, then $\underline{\underline{a p r}}{ }_{R}^{\mathcal{I}}(A)=\{b\} \subseteq\{b, c, d\}=\underline{\underline{a p r}}{ }_{R}^{\mathcal{I}}(B)$, but $A \nsubseteq B$.

(b) For part 7, take

1. $A=\{a, c, d\}$, then $\overline{\overline{a p r}}_{R}^{\mathcal{I}}(A)=A$, but $A \notin \mathcal{I}$.

2. $A=\{b\}$, then $\underline{\underline{a p r}}{ }_{R}^{\mathcal{I}}(A)=A$, but $A^{c} \notin \mathcal{I}$.

(c) For part 9, take

1. $A=\{a, c, d\}$, then $\overline{\overline{a p r}}_{R}^{\mathcal{I}}(A)=A$, but $\mathcal{I} \neq P(U)$.

2. $A=\{b\}$, then $\underline{\underline{a p r}}{ }_{R}^{\mathcal{I}}(A)=A$, but $\mathcal{I} \neq P(U)$.

(ii) Example 3.1 (ii) also shows that the converse of part 8 in Propositions 4.1 and 4.2 is not necessarily to be true in general. Take

1. $A=\{a, d\}$, then $\overline{\overline{a p r}}_{R}^{\mathcal{J}}(A)=\{a, d\} \subseteq\{a, d\}=\overline{\overline{a p r}}_{R}^{\mathcal{I}}(A)$, but $\mathcal{I} \nsubseteq \mathcal{J}$.

2. $A=\{b, c\}$, then $\underline{\underline{a p r}}{ }_{R}^{\mathcal{I}}(A)=\{b, c\} \subseteq\{b, c\}=\underline{\underline{a p r}}_{R}^{\mathcal{J}}(A)$, but $\mathcal{I} \nsubseteq \mathcal{J}$.

(iii) Example 3.1 (iii) also shows that the inclusion of parts 3 and 4 in Propositions 4.1 and 4.2 can not be replaced by the equality in general.

(a) For part 3, take

1. $A=\{b, c\}$, then $\overline{\overline{a p r}}_{R}^{\mathcal{I}}(A)=\{a, b, c\}, \overline{\overline{a p r}}_{R}^{\mathcal{I}}\left(\overline{\overline{a p r}}_{R}^{\mathcal{I}}(A)\right)=U$. Therefore, $\overline{\overline{a p r}}_{R}^{\mathcal{I}}(A)=$ $\{a, b, c\} \neq U=\overline{\overline{a p r}}_{R}^{\mathcal{I}}\left(\overline{\overline{a r r}}_{R}^{\mathcal{I}}(A)\right)$.

2. $A=\{a, d\}$, then $\left.\underline{\underline{a p r}}_{R}^{\mathcal{I}}(A)=\{d\}, \underline{\underline{a p r}}{ }_{R}^{\mathcal{I}} \underline{\underline{a p r}}{ }_{R}^{\mathcal{I}}(A)\right)=\phi$. Therefore, $\underline{\underline{a p r}}_{R}^{\mathcal{I}}(A)=$

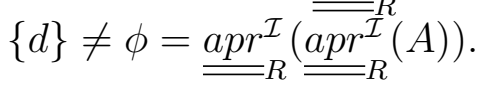

(b) For part 4, take

1. $A=\{a, d\}, B=\{b, c\}, A \cap B=\phi$. Hence, $\overline{\overline{a p r}}_{R}^{\mathcal{I}}(A)=U, \overline{\overline{a p r}}_{R}^{\mathcal{I}}(B)=\{a, b, c\}$. Therefore, $\overline{\overline{a p r}}_{R}^{\mathcal{I}}(A) \cap \overline{\overline{a p r}}_{R}^{\mathcal{I}}(B)=\{a, b, c\} \neq \phi=\overline{\overline{a p r}}_{R}^{\mathcal{I}}(A \cap B)$.

2. $A=\{a, d\}, B=\{b, c\}, A \cup B=U$. Hence, $\underline{\underline{\underline{a p r}}} R_{R}^{\mathcal{I}}(A)=\{d\}, \underline{\underline{a p r}}{ }_{R}^{\mathcal{I}}(B)=\phi$. Therefore, $\underline{\underline{\operatorname{apr}_{R}^{\mathcal{I}}}}(A) \cup \underline{\underline{\operatorname{apr}^{\mathcal{I}}}}(B)=\{d\} \neq U=\underline{\underline{\underline{a p r}_{R}^{\mathcal{I}}}}(A \cup B)$. 
Remark 4.2. There are some properties which are not hold or satisfied in the second type.

(i) In Example 3.1 (i) take

1. $A=\{a\} \in \mathcal{I}$, then $\overline{\overline{a p r}}_{R}^{\mathcal{I}}(A)=A$. Hence, if $A \in \mathcal{I} \nRightarrow \overline{\overline{a p r}}_{R}^{\mathcal{I}}(A)=\phi$

2. $A^{c}=\{a\} \in \mathcal{I}$, then $\underline{\underline{\operatorname{apr}^{\mathcal{I}}}}(A)=A$. Hence, if $A^{c} \in \mathcal{I} \nRightarrow \underline{\underline{\operatorname{apr}^{\mathcal{I}}}}(A)=U$.

(ii) In Example 3.1 (ii) take

1. $\mathcal{J}=P(U), A=\{a, d\}$, then $\overline{\overline{a p r}}_{R}^{\mathcal{J}}(A)=A$. Hence, if $\mathcal{J}=P(U) \nRightarrow \overline{\overline{a p r}}_{R}^{\mathcal{J}}(A)=\phi$.

2. $\mathcal{J}=P(U), A=\{b, c\}$, then $\underline{\underline{\operatorname{apr}^{\mathcal{J}}}}(A)=A$. Hence, if $\mathcal{J}=P(U) \nRightarrow \underline{\underline{a p r}}_{R}^{\mathcal{J}}(A)=U$.

(iii) In Example 3.2 take

1. $A=\{c\}$, then $\overline{\overline{a p r}}_{R}^{\mathcal{I}}(A)=\{a, b, c\}, \underline{\underline{a p r}}{ }_{R}^{\mathcal{I}}\left(\overline{\overline{a p r}}_{R}^{\mathcal{I}}(A)\right)=\{c\}$. Therefore, $\overline{\overline{a p r}}_{R}^{\mathcal{I}}(A)=$ $\{a, b, c\} \nsubseteq\{c\}=\underline{\underline{a p r}}{ }_{R}^{\mathcal{I}}\left(\overline{\overline{a p r}}_{R}^{\mathcal{I}}(A)\right)$.

2. $A=\{a, b, d\}$, then $\underline{\underline{a p r}}_{R}^{\mathcal{I}}(A)=\{d\}, \overline{\overline{a p r}}_{R}^{\mathcal{I}}\left(\underline{\underline{a p r}}_{R}^{\mathcal{I}}(A)\right)=\{a, b, d\}$. Therefore, $\overline{\overline{a p r}}_{R}^{\mathcal{I}}$ $\left.\underline{\underline{a p r}}_{R}^{\mathcal{I}}(A)\right)=\left\{a, b, \overline{\overline{d d}\}} \stackrel{R}{\nsubseteq}\{d\}=\underline{\underline{a p r}}_{R}^{\mathcal{I}}(A)\right.$.

The boundary of a subset decreases and the accuracy increases as the ideal on $U$ increases as it is shown in the following theorem.

Theorem 4.1. Let $A \subseteq U, \mathcal{I}, \mathcal{J}$ be two ideals on $U$ and $R$ be a binary relation on $U$. If $\mathcal{I} \subseteq \mathcal{J}$. Then,

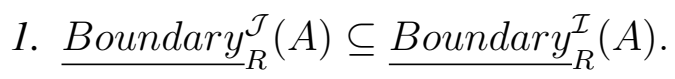

2. $\underline{\operatorname{Accuracy}}_{R}^{\mathcal{I}}(A) \leq \underline{\operatorname{Accuracy}}_{R}^{\mathcal{J}}(A)$.

Proof. Similar to the proof of Theorem 3.1.

Remark 4.3. Example 3.1 (ii) shows that the converse of parts 1 and 2 in Theorem 4.1 is not necessary to be true in general. Take, $A=\{b, c\}$, then

1. $\underline{\text { Boundary }}_{R}^{\mathcal{J}}(A)=\phi \subseteq\{a\}=\underline{\text { Boundary }}_{R}^{\mathcal{I}}(A)$, but $\mathcal{I} \nsubseteq \mathcal{J}$.

2. $\underline{\operatorname{Accuracy}}_{R}^{\mathcal{J}}(A)=1 \leq \frac{2}{3}=\underline{\operatorname{Accuracy}}_{R}^{\mathcal{I}}(A)$, but $\mathcal{I} \nsubseteq \mathcal{J}$.

Theorem 4.2. Let $A \subseteq U, \mathcal{I}$ be an ideal on $U$ and $R_{1}, R_{2}$ be two similarity relations on $U$. If $R_{1} \subseteq R_{2}$, then

1. $\overline{\overline{a p r}}_{R_{1}}^{\mathcal{I}}(A) \subseteq \overline{\overline{a p r}}_{R_{2}}^{\mathcal{I}}(A)$.

2. $\underset{\underline{a p r^{\mathcal{I}}}}{R_{2}}(A) \subseteq \underline{\underline{a p r^{\mathcal{I}}}} R_{1}(A)$. 
3. $\underline{\text { Boundary }}_{R_{1}}^{\mathcal{I}}(A) \subseteq \underline{\text { Boundary }}_{R_{2}}^{\mathcal{I}}(A)$.

4. $\underline{\text { Accuracy }}_{R_{2}}^{\mathcal{I}}(A) \leq \underline{\text { Accuracy }}_{R_{1}}^{\mathcal{I}}(A)$.

5. $\underline{\text { Roughness }}_{R_{1}}^{\mathcal{I}}(A) \leq \underline{\text { Roughness }} R_{2}^{\mathcal{I}}(A)$.

Proof. Similar to Theorem 3.2.

Remark 4.4. Example 3.3 shows that the inclusion and the less than in Theorem 4.2 can not be replaced by equality relation in general. Take $A=\{a, d\}$, then

1. $\overline{\overline{a p r}}_{R_{1}}^{\mathcal{I}}(A)=\{a, b, d\} \neq U=\overline{\overline{a p r}}_{R_{2}}^{\mathcal{I}}(A)$.

2. $\underline{\text { Boundary }}_{R_{1}}^{\mathcal{I}}(A)=\{b\} \neq\{b, c\}=\underline{\text { Boundary }}_{R_{2}}^{\mathcal{I}}(A)$.

3. $\underline{\text { Accuracy }}_{R_{1}}^{\mathcal{I}}(A)=\frac{2}{3} \neq \frac{1}{2}=\underline{\text { Accuracy }}_{R_{2}}^{\mathcal{I}}(A)$.

4. $\underline{\text { Roughness }}_{R_{1}}^{\mathcal{I}}(A)=0.3 \neq 0.5=\underline{\text { Roughness }_{R_{2}}^{\mathcal{I}}}(A)$.

The following theorem presents the relationships between the current approximations in Definitions 3.1 and 4.1 .

Theorem 4.3. Let $A \subseteq U, \mathcal{I}$ be an ideal on $U$ and $R$ be a binary relation on $U$. Then,

1. $\overline{\operatorname{apr}}_{R}^{\mathcal{I}}(A) \subseteq \overline{\overline{a p r}}_{R}^{\mathcal{I}}(A)$.

2. $\underline{\underline{\operatorname{apr}}}_{R}^{\mathcal{I}}(A) \subseteq \underline{\operatorname{apr}}_{R}^{\mathcal{I}}(A)$.

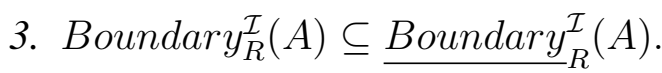

4. $\operatorname{Accuracy}_{R}^{\mathcal{I}}(A) \leq \operatorname{Accuracy}_{R}^{\mathcal{I}}(A)$.

Proof. Immediately by using the Definitions 3.1 and 4.1.

Remark 4.5. Example 3.3 shows that the inclusion and less than in Theorem 4.3 can not be replaced by equality relation in general. Take $A=\{a, c, d\}$, then

1. $\overline{\operatorname{apr}}_{R_{1}}^{\mathcal{I}}(A)=\{a, b\} \neq U=\overline{\overline{a p r}}_{R_{1}}^{\mathcal{I}}(A)$.

2. $\underset{\underline{a p r^{\mathcal{I}}}}{R_{1}}(A)=\{a, c, d\} \neq U=\underline{a p r}_{R_{1}}^{\mathcal{I}}(A)$.

3. Boundary $\operatorname{I}_{1}^{\mathcal{I}}(A)=\phi \neq\{b\}=\underline{B o u n d a r y}_{R_{1}}^{\mathcal{I}}(A)$.

4. $\underline{\text { Accuracy }}_{R_{1}}^{\mathcal{I}}(A)=\frac{3}{4} \lesseqgtr 2=\operatorname{Accuracy}_{R_{1}}^{\mathcal{I}}(A)$. 
Comparisons between the current approximations in Definition 4.1 and the previous one in Definition 2.4 [8] are given in the following theorem.

Theorem 4.4. Let $A \subseteq U, \mathcal{I}$ be an ideal on $U$ and $R$ be a similarity relation on a nonempty set U. Then,
1. $\overline{\overline{a p r}}_{R}^{\mathcal{I}}(A) \subseteq \overline{\operatorname{apr}}_{R}(A)$.
2. ${\underset{\operatorname{apr}}{R}}_{R}(A) \subseteq \underline{\operatorname{apr}}_{R}^{\mathcal{I}}(A)$.
3. Boundary $_{R}^{\mathcal{I}}(A) \subseteq$ Boundary $_{R}(A)$.

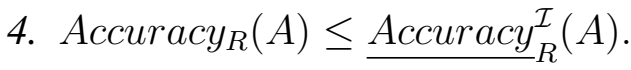
5. Roughness $_{R}^{\mathcal{I}}(A) \leq$ Roughness $_{R}(A)$.

Proof. The proof is similar to that of Theorem 3.3.

Remark 4.6. Example 3.3 shows that the inclusion and the less than in Theorem 4.4 can not be replaced by equality relation. Take $A=\{b, c, d\}$, then $\overline{\overline{a p r}}_{R_{1}}^{\mathcal{I}}(A)=\{b, c, d\} \neq U=\overline{a p r}_{R_{1}}(A)$. Moreover, if $A=\{a, c, d\}$, then

1. $\underline{\operatorname{apr}}_{R_{1}}(A)=\{c, d\} \neq\{a, c, d\}=\underline{\underline{a p r}}{ }_{R_{1}}^{\mathcal{I}}(A)$.

2. Boundary $_{R_{1}}^{\mathcal{I}}(A)=\{b\} \neq\{a, b\}=$ Boundary $_{R_{1}}(A)$.

3. $\operatorname{Accuracy}_{R_{1}}(A)=\frac{1}{2} \lesseqgtr \frac{3}{4}=\underline{\operatorname{Accuracy}}_{R_{1}}^{\mathcal{I}}(A)$.

4. $\underline{\text { Roughness }}_{R_{1}}^{\mathcal{I}}(A)=\frac{1}{4} \lesseqgtr \frac{1}{2}=$ Roughness $_{R_{1}}(A)$.

\section{The third method of generalized rough sets via ideals}

This section mainly concerns with the third type of generalized rough approximations which based on ideals and the union of neighborhoods which calculated by any binary relation. The fundamental characteristics of these approximations is interjected and analyzed. Additionally, an example is introduced to show that the corresponding lower and upper approximations, boundary regions, accuracy measures and roughness measures of this type of these approximations are not monotonic. After this, the comparisons between these approximations and the approximations in Sections 3 and 4 are introduced. Finally, the relationships between these approximations and the previous ones in [8] are discussed.

Definition 5.1. Let $R$ be a binary relation on a nonempty set $U$ and $\mathcal{I}$ be an ideal on $U$. For any subset $A \subseteq U$, the third kind of the generalized lower, upper approximations, boundary regions, accuracy and roughness of $A$ via ideal and according to $R$ is defined respectively by:

$\operatorname{apr}_{R}^{\prime \mathcal{I}}(A)=\cup\left\{<x>\breve{R}:<x>\breve{R} \cap A^{c} \in \mathcal{I}\right\}$. 
$\overline{\operatorname{apr}}_{R}^{\mathcal{I}}(A)=\left(\underline{\operatorname{apr}}_{R}^{\prime}\left(A^{c}\right)\right)^{c}$.

$\operatorname{Boundary}_{R}^{\prime \mathcal{I}} \overline{(A)}=\overline{\operatorname{apr}}_{R}^{\mathcal{I}}(A)-\underline{\operatorname{apr}}_{R}{ }_{R}^{\mathcal{I}}(A)$.

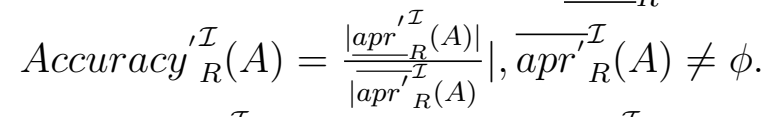

$\operatorname{Roughness}_{R}^{\prime \mathcal{I}}(A)=1-\operatorname{Accuracy}_{R}^{\prime \mathcal{I}}(A)$.

Proposition 5.1. Let $A, B \subseteq U, \mathcal{I}, \mathcal{J}$ be two ideals on $U$ and $R$ be a binary relation on $U$. Then, the following properties hold:

1. $A \subseteq B \Rightarrow \underline{a p r}_{R}^{\prime \mathcal{I}}(A) \subseteq \underline{a p r}_{R}^{\prime \mathcal{I}}(B)$.

2. $\underline{a p r}_{R}^{\prime \mathcal{I}}(A) \cup \underline{a p r}_{R}^{\prime \mathcal{I}}(B) \subseteq \underline{a p r}_{R}^{\prime \mathcal{I}}(A \cup B)$.

3. $\underline{\operatorname{apr}}_{R}^{\prime \mathcal{I}}(A \cap B) \subseteq \underline{a p r}_{R}^{\prime \mathcal{I}}(A) \cap \underline{a p r}_{R}^{\prime \mathcal{I}}(B)$.

4. ${\underset{\operatorname{apr}}{R}}_{R}^{\mathcal{I}}(A)=\left(\overline{\operatorname{apr}}_{R}^{I}{ }_{R}^{\mathcal{I}}\left(A^{c}\right)\right)^{c}$.

5. if $\mathcal{I} \subseteq \mathcal{J}$, then $\underline{\operatorname{apr}}_{R}^{\prime \mathcal{I}}(A) \subseteq \underline{\operatorname{apr}}_{R}^{\prime \mathcal{J}}(A)$.

6. $\underline{\operatorname{apr}}_{R}^{\prime \mathcal{I} \cap \mathcal{J}}(A)=\underline{a p r}_{R}^{\prime \mathcal{I}}(A) \cap \underline{a p r}_{R}^{\prime \mathcal{J}}(A)$.

\section{Proof.}

1. Let $A \subseteq B$ and $x \in \frac{a p r}{R}_{R}^{\prime \mathcal{I}}(A)$. Then, $\exists y \in U$ such that $x \in<y>\breve{R} \cap A^{c} \in \mathcal{I}$. Hence, $x \in<y>\breve{R} \cap B^{c} \in \mathcal{I}$ (by $B^{c} \subseteq A^{c}$, and the properties of ideal). Thus, $x \in \frac{a p r^{\prime}}{R}(B)$. Therefore, $\underline{\operatorname{apr}}_{R}^{\prime \mathcal{I}}(A) \subseteq{\underline{a p r^{\prime I}}}_{R}^{\mathcal{I}}(B)$.

2. Immediately by part (1).

3. Immediately by part (1).

4. Straightforward by Definition 5.1 .

5. Let $\mathcal{I} \subseteq \mathcal{J}$ and $x \in \underline{a p r}_{R}^{\prime \mathcal{I}}(A)$. Then, $\exists y \in U$ such that $x \in<y>\breve{R} \cap A^{c} \in \mathcal{I} \subseteq \mathcal{J}$. So, $x \in \underline{\operatorname{apr}}_{R}^{\prime \mathcal{J}}(A)$, and hence $\underline{\operatorname{apr}}_{R}^{\prime \mathcal{I}}(A) \subseteq \underline{\operatorname{apr}}_{R}^{\prime \mathcal{J}}(A)$.

6.

$$
\begin{aligned}
\underline{\operatorname{apr}}_{R}^{\prime \mathcal{I} \cap \mathcal{J}}(A) & =\cup\left\{<x>\breve{R}:<x>\breve{R} \cap A^{c} \in \mathcal{I} \cap \mathcal{J}\right\} . \\
& =\left(\cup\left\{<x>\breve{R}:<x>\breve{R} \cap A^{c} \in \mathcal{I}\right\}\right) \text { and }\left(\cup\left\{<x>\breve{R}:<x>\breve{R} \cap A^{c} \in \mathcal{J}\right\}\right) . \\
& =\left(\cup\left\{<x>\breve{R}:<x>\breve{R} \cap A^{c} \in \mathcal{I}\right\}\right) \cap\left(\cup\left\{<x>\breve{R}:<x>\breve{R} \cap A^{c} \in \mathcal{J}\right\}\right) . \\
& =\underline{\operatorname{apr}}_{R}^{\prime \mathcal{I}}(A) \cap \underline{\operatorname{apr}}_{R}^{\prime \mathcal{J}}(A) .
\end{aligned}
$$


Proposition 5.2. Let $A, B \subseteq U, \mathcal{I}, \mathcal{J}$ be two ideals on $U$ and $R$ be a binary relation on $U$. Then, the following properties hold:

1. $A \subseteq B \Rightarrow \overline{a p r}_{R}^{\mathcal{I}}(A) \subseteq \overline{\operatorname{apr}}_{R}^{\mathcal{I}}(B)$.

2. $\overline{\operatorname{apr}}_{R}^{\mathcal{I}}(A \cap B) \subseteq \overline{\operatorname{apr}}_{R}^{\mathcal{I}}(A) \cap \overline{\operatorname{apr}}_{R}^{\mathcal{I}}(B)$.

3. $\overline{\operatorname{apr}}_{R}^{\mathcal{I}}(A) \cup{\overline{a p r^{\prime}}}_{R}^{\mathcal{I}}(B) \subseteq{\overline{a p r^{\prime}}}_{R}^{\mathcal{I}}(A \cup B)$.

4. $\overline{\operatorname{apr}}_{R}^{\prime}{ }_{R}^{\mathcal{I}}(A)=\left(\underline{\operatorname{apr}}_{R}^{\prime \mathcal{I}}\left(A^{c}\right)\right)^{c}$.

5. if $\mathcal{I} \subseteq \mathcal{J}$, then $\overline{\operatorname{apr}}_{R}^{\mathcal{J}}(A) \subseteq \overline{\operatorname{apr}}_{R}^{\mathcal{I}}(A)$.

6. $\overline{\operatorname{apr}}_{R}^{\mathcal{I} \cap \mathcal{J}}(A)=\overline{\operatorname{apr}}_{R}^{\mathcal{I}}(A) \cup{\overline{a p r^{\prime}}}_{R}^{\mathcal{J}}(A)$.

\section{Proof.}

1. Let $A \subseteq B$. Thus, $B^{c} \subseteq A^{c}$, and hence $\frac{a p r^{\prime I}}{R}\left(B^{c}\right) \subseteq \underline{a p r}_{R}^{\prime I}\left(A^{c}\right)$ (by No. (1) in Proposition 5.1). So, $\left(\underline{a p r}_{R}^{\prime \mathcal{I}}\left(A^{c}\right)\right)^{c} \subseteq\left(\underline{a p r}_{R}^{\prime \mathcal{I}}\left(B^{c}\right)\right)^{c}$. Consequently, ${\overline{a p r^{\prime}}}_{R}^{\mathcal{I}}(A) \subseteq{\overline{a p r^{\prime}}}_{R}^{\mathcal{I}}(B)$.

2. Immediately by part (1).

3. Immediately by part (1).

4. Straightforward by Definition 5.1 .

5. Let $\mathcal{I} \subseteq \mathcal{J}$ and $x \in{\overline{a p r^{\prime}}}_{R}^{\mathcal{J}}(A)$. Then, $\left.x \in \underline{(a p r}_{R}^{\mathcal{J}}\left(A^{c}\right)\right)^{c} \subseteq\left(\underline{a p r}_{R}^{\prime \mathcal{I}}\left(A^{c}\right)\right)^{c}$, (by No. (5) in Proposition 5.1). Thus, $x \in\left(\underline{a p r}_{R}^{\prime \mathcal{I}}\left(A^{c}\right)\right)^{c}={\overline{a p r^{\prime}}}_{R}^{\mathcal{I}}(A)$. Therefore, $\overline{\operatorname{apr}^{\prime}}{ }_{R} \mathcal{J}(A) \subseteq{\overline{a p r^{\prime}}}_{R}^{\mathcal{I}}(A)$.

6.

$$
\begin{aligned}
& {\overline{a p r^{\prime}}}_{R}^{\mathcal{I} \cap \mathcal{J}}(A)=\left(\underline{\operatorname{apr}}_{R}^{\prime \mathcal{I} \cap \mathcal{J}}\left(A^{c}\right)\right)^{c} . \\
& =\left(\underline{\operatorname{apr}}_{R}^{\prime \mathcal{I}}\left(A^{c}\right) \cap \underline{\operatorname{apr}}_{R}^{\prime \mathcal{J}}\left(A^{c}\right)\right)^{c}(\text { by No. (6) in Proposition 5.1). } \\
& =\left(\underline{\operatorname{apr}}_{R}^{\mathcal{I}}\left(A^{c}\right)\right)^{c} \cup\left(\underline{a p r}_{R}^{\mathcal{J}}\left(A^{c}\right)\right)^{c} \text {. } \\
& ={\overline{a p r^{\prime}}}_{R}^{\mathcal{I}}(A) \cup{\overline{a p r^{\prime}}}_{R}^{\mathcal{J}}(A) \text {. }
\end{aligned}
$$

Remark 5.1. 1. Example 3.1 (i) shows that the converse of part 1 in Propositions 5.1 and 5.2 is not necessarily to be true in general. Take

(a) $A=\{a\}, B=\{d\}$, then ${\overline{a p r^{\prime}}}_{R}^{\mathcal{I}}(A)=\phi,{\overline{a p r^{\prime}}}_{R}^{\mathcal{I}}(B)=\{a, d\}$. Therefore, ${\overline{a p r^{\prime}}}_{R}^{\mathcal{I}}(A) \subseteq$ $\overline{\operatorname{apr}}_{R}^{\prime}(B)$, but $A \nsubseteq B$.

(b) $A=\{b\}, B=\{a, c, d\}$, then $\underline{a p r}_{R}^{\prime \mathcal{I}}(A)=\{b, c\}, \underline{a p r}_{R}^{\prime \mathcal{I}}(B)=U$. Therefore, $\underline{a p r}_{R}^{\prime \mathcal{I}}(A) \subseteq$ $\underline{\operatorname{apr}}_{R}^{\prime \mathcal{I}}(B)$, but $A \nsubseteq B$. 
2. Example 3.1 (iii) shows that the inclusion of part 2 in Propositions 5.1 and 5.2 can not be replaced by equality relation in general. Take $A=\{a, d\}, B=\{b, c\}$, then

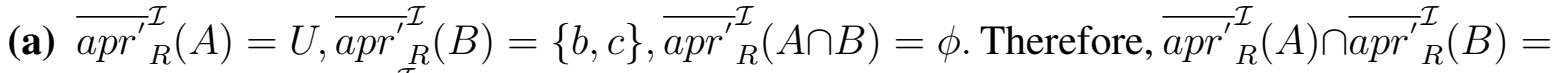 $\{b, c\} \neq \phi={\overline{a p r^{\prime}}}_{R}^{\mathcal{I}}(A \cap B)$.

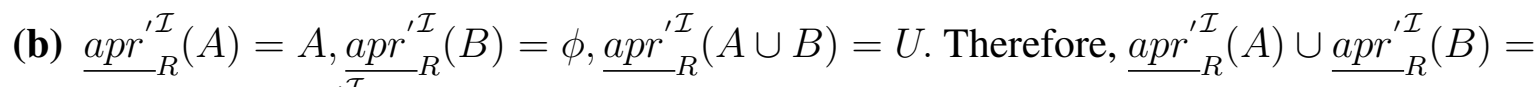 $A \neq U=\underline{a p r}_{R} \bar{I}(A \cup B)$.

3.

Example 5.1. Let $U=\{a, b, c, d\}, \mathcal{I}=\{\phi,\{a\}\}$ and $R=\{(a, a),(a, c),(a, d),(b, a),(b, b)$, $(b, c),(d, a),(d, b)\}$ be a binary relation defined on $U$ thus $<a>\breve{R}=<c>\breve{R}=U,<$ $b>\breve{R}=\{a, b, c\},<d>\breve{R}=\{a, c, d\}$. This example shows that the inclusion of part 3 in Propositions 5.1 and 5.2 can not be replaced by equality relation in general. Take

(a) $A=\{a, c, d\}, B=\{a, b, c\}, A \cap B=\{a, c\}$, then $\frac{a p r^{I \mathcal{I}}}{R}(A)=A,{\frac{a p r}{{ }^{I}}}^{I}(B)=$ $B,{ }_{a p r}^{\prime \mathcal{I}}(A \cap B)=\phi$. Therefore, $\underline{a p r}_{R}^{\prime \mathcal{I}}(A) \cap \underline{a p r}_{R}^{\prime \mathcal{I}}(B)=\{a, c\} \neq \phi={\underline{a p r^{\prime \mathcal{I}}}}_{R}^{R}(A \cap B)$.

(b) $A=\{b\}, B=\{d\}, A \cup B=\{b, d\}$, then ${\overline{a p r^{\prime}}}_{R}^{\mathcal{I}}(A)=A,{\overline{a p r^{\prime}}}_{R}^{\mathcal{I}}(B)=B,{\overline{a p r^{\prime}}}_{R}^{\mathcal{I}}(A \cup$ $B)=U$. Therefore, ${\overline{a p r^{\prime}}}_{R}^{\mathcal{I}}(A) \cup{\overline{a p r^{\prime}}}_{R}^{\mathcal{I}}(B)=\{b, d\} \neq U={\overline{a p r^{\prime}}}_{R}^{\mathcal{I}}(A \cup B)$.

4. Example 3.1 (ii) shows that the converse of part 5 in Propositions 5.1 and 5.2 is not necessarily to be true in general. Take

(a) $A=\{a, d\}$, then ${\overline{a p r^{\prime}}}_{R}^{\mathcal{I}}(A)=\{a, d\},{\overline{a p r^{\prime}}}_{R}^{\mathcal{J}}(A)=\{d\}$. Therefore, ${\overline{a p r^{\prime}}}_{R}^{\mathcal{J}}(A) \subseteq{\overline{a p r^{\prime}}}_{R}^{\mathcal{I}}(A)$, but $\mathcal{I} \nsubseteq \mathcal{J}$.

(b) $A=\{b, c\}$, then $\underline{a p r}_{R}^{\prime \mathcal{I}}(A)=\{b, c\}, \underline{a p r}_{R}^{\prime \mathcal{J}}(A)=\{a, b, c\}$. Therefore, $\underline{a p r}_{R}^{\prime \mathcal{I}}(A) \subseteq$ $\operatorname{apr}_{R}^{\prime \mathcal{J}}(A)$, but $\mathcal{I} \nsubseteq \mathcal{J}$.

Remark 5.2. There are some properties which are not hold or satisfied in the third type.

(i) In Example 3.1 (i) take
1. $A=\{a\}$, then ${\overline{a p r^{\prime}}}_{R}^{\mathcal{I}}(A)=\phi$. Hence, $A \nsubseteq{\overline{a p r^{\prime}}}_{R}^{\mathcal{I}}(A)$.
2. $A=\{b, c, d\}$, then $\underline{a p r}_{R}^{\prime \mathcal{I}}(A)=U$. Hence, $\underline{a p r}_{R}^{\prime \mathcal{I}}(A) \nsubseteq A$.
3. $A=U$, then ${\overline{a p r^{\prime}}}_{R}^{\mathcal{I}}(U)=\{a, d\}$. Hence, ${\overline{a p r^{\prime}}}_{R}^{\mathcal{I}}(U) \neq U$.
4. $A=\phi$, then $\underline{a p r}_{R}^{\prime \mathcal{I}}(\phi)=\{b, c\}$. Hence, $\underline{a p r}_{R}^{\prime \mathcal{I}}(\phi) \neq \phi$.

(ii)

Example 5.2. Let $U=\{a, b, c, d\}, \mathcal{I}=\{\phi,\{a\}\}$ and $R=\{(a, a)\}$ be a binary relation defined on $U$ thus $<a>\breve{R}=\{a\},<b>\breve{R}=<c>\breve{R}=<d>\breve{R}=\phi$. Take 
1. $A=U$, then $\underline{\operatorname{apr}}_{R}^{\prime \mathcal{I}}(U)=\{a\}$. Hence, $\underline{\operatorname{apr}}_{R}^{\prime \mathcal{I}}(U) \neq U$.

2. $A=\phi$, then $\overline{\operatorname{apr}}_{R}^{\mathcal{I}}(\phi)=\{b, c, d\}$. Hence, $\overline{\operatorname{apr}}_{R}^{\mathcal{I}}(\phi) \neq \phi$.

(iii) In Example 5.2 take

1. $A=\{b, c, d\}$, then $A^{c} \in \mathcal{I}$, then $\underline{\operatorname{apr}}_{R}^{\prime \mathcal{I}}(A)=\{a\}$. Hence, if $A^{c} \in \mathcal{I} \nRightarrow \underline{a p r}_{R}^{\prime \mathcal{I}}(A)=U$ or $A$.

2. $A=\{a\} \in \mathcal{I}$, then ${\overline{a p r^{\prime}}}_{R}^{\mathcal{I}}(A)=\{b, c, d\}$. Hence, if $A \in \mathcal{I} \nRightarrow{\overline{a p r^{\prime}}}_{R}^{\mathcal{I}}(A)=\phi$ or $A$.

3. $A=\{b, c, d\}, \mathcal{I}=P(U)$, then $\underline{a p r}_{R}^{\prime \mathcal{I}}(A)=\{a\}$. Hence, if $\mathcal{I}=P(U) \nRightarrow \underline{a p r}_{R}^{\prime \mathcal{I}}(A)=$ $U$, or $A$.

4. $A=\{a\}, \mathcal{I}=P(U)$, then ${\overline{a p r^{\prime}}}_{R}^{\mathcal{I}}(A)=\{b, c, d\}$. Hence, if $\mathcal{I}=P(U) \nRightarrow \overline{a p r}_{R}^{\mathcal{I}}(A)=$ $\phi$, or $A$.

The following theorem shows that the boundary of a subset decreases and the accuracy increases as the ideal on $U$ increases.

Theorem 5.1. Let $A \subseteq U, \mathcal{I}, \mathcal{J}$ be two ideals on $U$ and $R$ be a binary relation on $U$. If $\mathcal{I} \subseteq \mathcal{J}$. Then,

1. Boundary ${ }_{R}^{\prime}(A) \subseteq$ Boundary $_{R}^{\prime \mathcal{I}}(A)$.

2. $\operatorname{Accuracy}_{R}^{\prime \mathcal{I}}(A) \leq \operatorname{Accuracy}_{R}^{\prime \mathcal{J}}(A)$.

Proof. Similar to Theorem 3.1.

Remark 5.3. Example 3.1 (ii) shows that the converse of parts 1 and 2 in Theorem 5.1 is not necessary to be true in general. Take, $A=\{b, c\}$, then

1. Boundary ${ }_{R}^{\prime}(A)=\{d\} \subseteq\{d\}=$ Boundary $_{R}^{\prime \mathcal{I}}(A)$, but $\mathcal{I} \nsubseteq \mathcal{J}$.

2. $\operatorname{Accuracy}_{R}^{\prime \mathcal{I}}(A)=\frac{2}{3}<1=\operatorname{Accuracy}_{R}^{\prime \mathcal{J}}(A)$, but $\mathcal{I} \nsubseteq \mathcal{J}$.

The following example shows that the corresponding lower and upper approximations, boundary regions, accuracy measures, roughness measures in third type does not have the monotonicity.

Example 5.3. Let $U=\{a, b, c, d, e, f, g\}, \mathcal{I}=\{\phi,\{a\}\}, R_{1}, R_{2}$ be similarity relations on $U$ where $R_{1}=\Delta \cup\{(a, c),(c, a),(c, g),(d, f),(e, g),(f, d),(g, c),(g, e)\}, R_{2}=\Delta \cup\{(a, c),(a, d)$, $(a, e),(b, f),(c, a),(c, g),(d, a),(d, f),(e, a),(e, g),(f, b),(f, d),(g, c),(g, e)\}$. Thus, $<a>\breve{R}_{1}$ $=\{a, c, g\},<b>\breve{R}_{1}=\{b\},<c>\breve{R}_{1}=<g>\breve{R}_{1}=\{a, c, e, g\},<d>\breve{R}_{1}=<f>\breve{R}_{1}=$ $\{d, f\},<e>\breve{R}_{1}=\{c, e, g\},<a>\breve{R}_{2}=\{a, c, d, e, f, g\},<b>\breve{R}_{2}=\{b, d, f\},<c>\breve{R}_{2}=<$ $e>\breve{R}_{2}=\{a, c, d, e, g\},<d>\breve{R}_{2}=\{a, b, c, d, e, f\},<f>\breve{R}_{2}=\{a, b, d, f\},<g>\breve{R}_{2}=$ $\{a, c, e, g\}$. Take 
1. $A=\{a, b, c, d, e, f\}$, then $\underline{a p r}_{R_{1}}^{\prime \mathcal{I}}(A)=\{b, d, f\}, \underline{a p r}_{R_{2}}^{\text {I }}(A)=A$. Therefore, $\underline{a p r}_{R_{1}}^{\prime \mathcal{I}}(A) \nsupseteq$ $\underline{\operatorname{apr}}_{R_{2}}^{\prime \mathcal{I}}(A)$.

2. $A=\{g\}$, then ${\overline{a p r^{\prime}}}_{R_{1}}^{\mathcal{I}}(A)=\{a, c, e, g\},{\overline{a p r^{\prime}}}_{R_{2}}^{\mathcal{I}}(A)=\{g\}$. Therefore, ${\overline{a p r^{\prime}}}_{R_{1}}^{\mathcal{I}}(A) \nsubseteq{\overline{a p r^{\prime}}}_{R_{2}}^{\mathcal{I}}(A)$.

3. $A=\{a, b, c, d, e, f\}$, then $\underline{a p r}_{R_{1}}^{\mathcal{I}}(A)=\{b, d, f\}, \overline{a p r}_{R_{1}}^{\mathcal{I}}(A)=U, \underline{a p r}_{R_{2}}^{\mathcal{I}_{2}}(A)=A, \overline{a p r}_{R_{2}}^{\mathcal{I}}(A)$ $=U$. Therefore,

(a) Boundary $_{R_{1}}^{\prime \mathcal{I}}(A)=\{a, c, e, g\} \nsubseteq\{g\}=$ Boundary $_{R_{2}}^{\prime \mathcal{I}}(A)$.

(b) Accuracy $_{R_{1}}^{\text {II }}(A)=\frac{3}{7}<\frac{6}{7}=\operatorname{Accuracy}_{R_{2}}^{\prime \mathcal{I}}(A)$.

(c) Roughness ${ }_{R_{1}}^{\prime \text { I }}(A)=\frac{4}{7}>\frac{1}{7}=$ Roughness $_{R_{2}}^{\prime \text { I }}(A)$.

Although, $R_{1} \subseteq R_{2}$.

The following theorem presents the relationships between the current approximations in Definitions 3.1, 4.1 and 5.1.

Theorem 5.2. Let $A \subseteq U, \mathcal{I}$ be an ideal on $U$ and $R$ be a reflexive relation on $U$. Then,

1. $\underline{\underline{a p r}}_{R}^{\mathcal{I}}(A) \subseteq \underline{a p r}_{R}^{\mathcal{I}}(A) \subseteq \underline{a p r}_{R}^{\prime \mathcal{I}}(A) \subseteq A \subseteq \overline{\operatorname{apr}}_{R}^{\mathcal{I}}(A) \subseteq \overline{\operatorname{apr}}_{R}^{\mathcal{I}}(A) \subseteq \overline{\overline{a p r}}_{R}^{\mathcal{I}}(A)$.

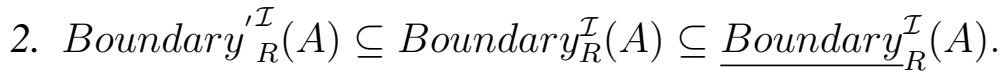

3. $\operatorname{Accuracy}_{R}^{\mathcal{I}}(A) \leq \operatorname{Accuracy}_{R}^{\mathcal{I}}(A) \leq \operatorname{Accuracy}_{R}^{\mathcal{I}^{\mathcal{I}}}(A)$.

Proof. We prove (1) only and the others are straightforward from (1). By Theorem 4.3, we have $\underline{a p r}_{R}^{\mathcal{I}}(A) \subseteq \underline{a p r} \underline{R}_{R}^{\mathcal{I}}(A)$. To prove, $\underline{a p r}_{R}^{\mathcal{I}}(A) \subseteq \underline{a p r}_{R}^{\prime \mathcal{I}}(A)$. Let $x \in \underline{a p r}_{R}^{\mathcal{I}}(A)$, then $<x>\breve{R} \cap A^{c} \in \mathcal{I}$. Hence, $\left\langle x>\breve{R} \subseteq \operatorname{apr}_{R}^{\prime \mathcal{I}}(A)\right.$. Since, $R$ is a reflexive relation, thus $x \in<x>\breve{R} \subseteq \operatorname{apr}_{R}^{\prime \mathcal{I}}(A)$. Therefore, $x \in \underline{a p r}_{R}^{\prime I}(A)$. Since, $R$ is reflexive, then $\underline{a p r}_{R}^{\prime I}(A) \subseteq A \subseteq{\overline{a p r^{\prime}}}_{R}^{\mathcal{I}}(A)$ straightforward from Definition 5.1. To prove, $\overline{a p r}_{R}^{\mathcal{I}}(A) \subseteq \overline{a p r}_{R}^{\mathcal{I}}(A)$. Let $x \in{\overline{a p r^{\prime}}}_{R}^{\mathcal{I}}(A)=\left(\underline{a p r}_{R}{ }_{R}\left(A^{c}\right)\right)^{c}$, then $x \notin \underset{a p r^{\prime}}{ }{ }_{R}\left(A^{c}\right)$. Hence, by Definition 5.1, we get $<x>\breve{R} \cap A \notin \mathcal{I}$. It follows that $x \in \overline{a p r}{ }_{R}^{\mathcal{I}}(A)$. By Theorem 4.3, we have $\overline{a p r} r_{R}^{\mathcal{I}}(A) \subseteq \overline{\overline{a p r}}_{R}^{\mathcal{I}}(A)$.

Remark 5.4. Example 3.1 (iii) shows that the inclusion and less than in Theorem 5.2 can not be replaced by equality relation in general. Take $A=\{b, c\}$, then $\overline{\operatorname{apr}}_{R}^{\mathcal{I}}(A)=\{b, c\} \varsubsetneqq\{a, b, c\}=$ $\overline{a p r}_{R}^{\mathcal{I}}(A)$. Moreover, Take $A=\{a, d\}$, then

1. $\underline{a p r}_{R}^{\mathcal{I}}(A)=\{d\} \varsubsetneqq\{a, d\}=\underline{a p r}_{R}^{\prime \mathcal{I}}(A)$.

2. Boundary' ${ }_{R}^{\mathcal{I}}(A)=\{b, c\} \varsubsetneqq\{a, b, c\}=\operatorname{Boundary}_{R}^{\mathcal{I}}(A)$.

3. $\operatorname{Accuracy}_{R}^{\mathcal{I}}(A)=\frac{1}{4} \lesseqgtr \frac{1}{2}=\operatorname{Accuracy}_{R}^{\prime \mathcal{I}}(A)$. 
The following theorem shows that the current approximations in Definition 5.1 is an extension and a generalization of and the previous Definition 2.5 [8].

Theorem 5.3. Let $A \subseteq U, \mathcal{I}$ be an ideal on $U$ and $R$ be a similarity relation on a nonempty set U. Then,
1. $\overline{\operatorname{apr}}_{R}^{\mathcal{I}}(A) \subseteq{\overline{a p r^{\prime}}}_{R}(A)$.
2. $\underline{\operatorname{apr}}_{R}^{\prime}(A) \subseteq \underline{\operatorname{apr}}_{R}^{\prime \mathcal{I}}(A)$.
3. Boundary $_{R}^{\prime \mathcal{I}}(A) \subseteq$ Boundary $_{R}^{\prime}(A)$.
4. $\operatorname{Accuracy}_{R}^{\prime}(A) \leq \operatorname{Accuracy}_{R}^{\prime \mathcal{I}}(A)$.

Proof. The proof is similar to that of Theorem 3.3.

Remark 5.5. Example 3.3 shows that the inclusion and less than in Theorem 5.3 can not be replaced by equality relation in general. Take $A=\{a, c, d\}$, then

1. ${\overline{a p r^{\prime}}}_{R_{1}}^{\mathcal{I}}(A)=\{a, b\} \neq U=\overline{a p r}_{R_{1}}(A)$.
2. ${\underline{a p r^{\prime}}}_{R_{1}}(A)=\{c, d\} \neq U=\underline{a p r}_{R_{1}}^{\prime}(A)$.

3. Boundary $_{R_{1}}^{\prime \mathcal{I}}(A)=\phi \neq\{a, b\}=$ Boundary $_{R_{1}}(A)$.

4. $\operatorname{Accuracy}_{R_{1}}^{\prime}(A)=\frac{1}{2} \lesseqgtr 2=\operatorname{Accuracy}_{R_{1}}^{\prime \mathcal{I}}(A)$.

\section{The fourth method of generalized rough sets via ideals}

The goal of this section is to define the fourth type of generalized rough approximations which depend on ideals and the union of neighborhoods which generated by any binary relation. The basic properties of these approximations is constructed and established. Moreover, the comparisons between these approximations and the approximations in Sections 3, 4 and 5 are illustrated. At the end of this section, the relationships between these approximations and the approximations in [8] are presented.

Definition 6.1. Let $R$ be a binary relation on a nonempty set $U$ and $\mathcal{I}$ be an ideal on $U$. For any subset $A \subseteq U$, the fourth kind of the generalized upper and lower approximations, boundary regions, accuracy and roughness of $A$ via ideal and according to $R$ is defined respectively by:

${\overline{a p r^{\prime \prime}}}_{R}(A)=\cup\{<x>\breve{R}:<x>\breve{R} \cap A \notin \mathcal{I}\}$.

$\underset{\operatorname{apr}_{R}^{\prime \prime}}{R}(A)=\left(\overline{\operatorname{apr}}_{R}^{\prime \prime}\left(A^{c}\right)\right)^{c}$.

$\operatorname{Boundary}_{R}^{\prime \prime \mathcal{I}}(A)=\overline{\operatorname{apr}}_{R}{ }_{R}^{\mathcal{I}}(A)-\underline{a p r}_{R}^{\prime I^{\mathcal{I}}}(A)$.

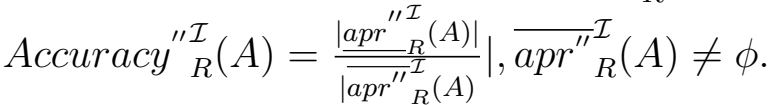

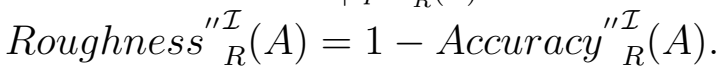


Proposition 6.1. Let $A, B \subseteq U, \mathcal{I}, \mathcal{J}$ be two ideals on $U$ and $R$ be a binary relation on $U$. Then, the following properties hold:

1. $\overline{\operatorname{apr}_{R}^{\prime \prime}}{ }_{R}^{\mathcal{I}}(\phi)=\phi$.

2. $A \subseteq B \Rightarrow{\overline{a p r^{\prime \prime}}}_{R}^{\mathcal{I}}(A) \subseteq \overline{\operatorname{apr}}_{R}{ }_{R}^{\mathcal{I}}(B)$.

3. $\overline{\operatorname{apr}}_{R}{ }_{R}^{\mathcal{I}}(A \cap B) \subseteq \overline{\operatorname{apr}}_{R}{ }_{R}^{\mathcal{I}}(A) \cap \overline{\operatorname{apr}}_{R}{ }_{R}^{\mathcal{I}}(B)$.

4. $\overline{\operatorname{apr}}_{R}{ }_{R}^{\mathcal{I}}(A \cup B)={\overline{a p r^{\prime \prime}}}_{R}^{\mathcal{I}}(A) \cup{\overline{a p r^{\prime \prime}}}_{R}^{\mathcal{I}}(B)$.

5. $\overline{\operatorname{apr}}_{R}^{\prime \prime} \mathcal{I}(A)=\left(\underline{\operatorname{apr}}_{R}^{\prime \prime} \mathcal{I}\left(A^{c}\right)\right)^{c}$.

6. if $A \in \mathcal{I}$, then $\overline{\operatorname{apr}^{\prime \prime}}{ }_{R}^{\mathcal{I}}(A)=\phi$.

7. if $\mathcal{I} \subseteq \mathcal{J}$, then $\overline{\operatorname{apr}}_{R}^{\prime} \mathcal{J}(A) \subseteq \overline{\operatorname{apr}}_{R}^{\mathcal{I}}(A)$.

8. if $\mathcal{I}=P(U)$, then $\overline{\operatorname{apr}^{\prime \prime}}{ }_{R}^{\mathcal{I}}(A)=\phi$.

9. $\overline{\operatorname{apr}}_{R}^{\mathcal{I} \cap \mathcal{J}}(A)={\overline{a p r^{\prime \prime}}}_{R}^{\mathcal{I}}(A) \cup{\overline{a p r^{\prime \prime}}}_{R}^{\mathcal{J}}(A)$.

10. $\overline{\operatorname{apr}}_{R}^{\mathcal{I} \vee \mathcal{J}}(A)={\overline{a p r^{\prime \prime}}}_{R}^{\mathcal{I}}(A) \cap \overline{\operatorname{apr}}_{R}^{\prime \prime} \mathcal{J}(A)$.

\section{Proof.}

1. $\overline{\operatorname{apr}^{\prime \prime}}{ }_{R}^{\mathcal{I}}(\phi)=\cup\{<x>\breve{R}:<x>\breve{R} \cap \phi \notin \mathcal{I}\}=\phi$.

2. Let $A \subseteq B$ and $x \in \overline{\operatorname{apr}^{\prime \prime}}{ }_{R}^{\mathcal{I}}(A)$. Then, $\exists y \in U$ such that $x \in<y>\breve{R}$ and $<y>\breve{R} \cap A \notin \mathcal{I}$. Thus, $<y>\breve{R} \cap B \notin \mathcal{I}$. So, $x \in{\overline{a p r^{\prime \prime}}}_{R}^{\mathcal{I}}(B)$. Consequently, $\overline{\operatorname{apr}}_{R} \mathcal{I}(A) \subseteq{\overline{a p r^{\prime \prime}}}_{R}^{\mathcal{I}}(B)$.

3. Immediately by part (2).

4.

$$
\begin{aligned}
& {\overline{a p r^{\prime \prime}}}_{R}^{\mathcal{I}}(A \cup B)=\cup\{<x>\breve{R}:<x>\breve{R} \cap(A \cup B) \notin \mathcal{I}\} . \\
& =(\cup\{<x>\breve{R}:<x>\breve{R} \cap A \notin \mathcal{I}\}) \cup(\cup\{<x>\breve{R}:<x>\breve{R} \cap B \notin \mathcal{I}\}) . \\
& =(\cup\{<x>\breve{R}:<x>\breve{R} \cap A \notin \mathcal{I}\}) \text { or }(\cup\{<x>\breve{R}:<x>\breve{R} \cap B \notin \mathcal{I}\}) \text {. } \\
& ={\overline{a p r^{\prime \prime}}}_{R}^{\mathcal{I}}(A) \cup{\overline{a p r^{\prime \prime}}}_{R}^{\mathcal{I}}(B) \text {. }
\end{aligned}
$$

5.

$$
\begin{aligned}
& \underbrace{(\operatorname{apr}}_{R}{ }_{R}^{\prime \mathcal{I}}\left(A^{c}\right))^{c}=\left(\left({\overline{a p r^{\prime \prime}}}_{R}^{\mathcal{I}}(A)\right)^{c}\right)^{c} . \\
& =\overline{\operatorname{apr}}_{R}^{\prime \prime} \mathcal{I}(A) \text {. }
\end{aligned}
$$




\section{Straightforward by Definition 6.1.}

7. Let $\mathcal{I} \subseteq \mathcal{J}, x \in{\overline{a p r^{\prime \prime}}}_{R}^{\mathcal{J}}(A)$. Then, $\exists y \in U$ such that $x \in<y>\breve{R}$ and $<y>\breve{R} \cap A \notin \mathcal{J}$. Thus, $\left\langle y>\breve{R} \cap A \notin \mathcal{I}\right.$ as $\mathcal{I} \subseteq \mathcal{J}$. So, $x \in{\overline{a p r^{\prime \prime}}}_{R}^{\mathcal{I}}(A)$. Hence, $\overline{\operatorname{apr}^{\prime \prime}}{ }_{R} \mathcal{J}(A) \subseteq{\overline{a p r^{\prime \prime}}}_{R}^{\mathcal{I}}(A)$.

\section{Straightforward by Definition 6.1.}

9.

$$
\begin{aligned}
& \overline{\operatorname{apr}}_{R}^{\prime \prime} \mathcal{I} \cap \mathcal{J}(A)=\cup\{<x>\breve{R}:<x>\breve{R} \cap A \notin \mathcal{I} \cap \mathcal{J}\} . \\
& =(\cup\{<x>\breve{R}:<x>\breve{R} \cap A \notin \mathcal{I}\}) \text { or }(\cup\{<x>\breve{R}:<x>\breve{R} \cap A \notin \mathcal{J}\}) \text {. } \\
& =(\cup\{<x>\breve{R}:<x>\breve{R} \cap A \notin \mathcal{I}\}) \cup(\cup\{<x>\breve{R}:<x>\breve{R} \cap A \notin \mathcal{J}\}) \text {. } \\
& =\overline{\operatorname{apr}}_{R} \mathcal{I}(A) \cup \overline{\operatorname{apr}_{R}^{\prime \prime}} \mathcal{J}(A) \text {. }
\end{aligned}
$$

10.

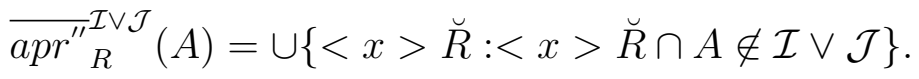

$$
\begin{aligned}
& =\cup\{<x>\breve{R}:<x>\breve{R} \cap A \notin \mathcal{I} \cup \mathcal{J}\} \text {. } \\
& =(\cup\{<x>\breve{R}:<x>\breve{R} \cap A \notin \mathcal{I}\}) \text { and }(\cup\{<x>\breve{R}:<x>\breve{R} \cap A \notin \mathcal{J}\}) \text {. } \\
& =(\cup\{<x>\breve{R}:<x>\breve{R} \cap A \notin \mathcal{I}\}) \cap(\cup\{<x>\breve{R}:<x>\breve{R} \cap A \notin \mathcal{J}\}) . \\
& =\overline{\operatorname{apr}}_{R}{ }_{R}^{\mathcal{I}}(A) \cap \overline{\operatorname{apr}}_{R}^{\prime \prime} \mathcal{J}(A) \text {. }
\end{aligned}
$$

Proposition 6.2. Let $A, B \subseteq U, \mathcal{I}, \mathcal{J}$ be two ideals on $U$ and $R$ be a binary relation on $U$. Then, the following properties hold:

1. $\underline{\operatorname{apr}}_{R}^{\prime \prime I}(U)=U$.

2. $A \subseteq B \Rightarrow \underline{a p r}_{R}^{\prime \prime I}(A) \subseteq \frac{a p r}{\prime \prime I}_{R}^{\mathcal{I}}(B)$.

3. $\frac{\operatorname{apr}}{\text { "II }}_{R}(A) \cup \underline{\operatorname{apr}}_{R}^{\prime \prime I}(B) \subseteq \underline{\operatorname{apr}}_{R}^{\prime \prime I}(A \cup B)$.

4. ${\underline{a p r^{\prime \prime I}}}_{R}(A \cap B)={\underline{a p r^{\prime \prime I}}}_{R}(A) \cap{\underline{a p r^{\prime \prime I}}}_{R}(B)$.

5. ${\underset{\operatorname{apr}}{ }}_{R}{ }_{R}^{\mathcal{I}}(A)=\left({\overline{a p r^{\prime \prime}}}_{R}^{\mathcal{I}}\left(A^{c}\right)\right)^{c}$.

6. if $A^{c} \in \mathcal{I}$, then $\underline{\operatorname{apr}}_{R}^{\prime \prime I}(A)=U$.

7. if $\mathcal{I} \subseteq \mathcal{J}$, then $\underline{\operatorname{apr}}_{R}^{\prime \prime \mathcal{I}}(A) \subseteq \underline{a p r}_{R}^{\prime \prime}(A)$.

8. if $\mathcal{I}=P(U)$, then $\underline{\operatorname{apr}}_{R}^{\prime \prime I}(A)=U$. 


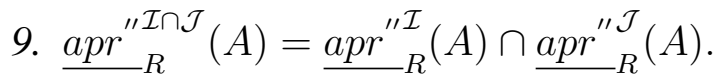

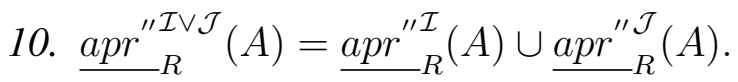

\section{Proof.}

1. ${ }_{\operatorname{apr}}{ }_{R}^{\prime \mathcal{I}}(U)=\left({\overline{a p r^{\prime \prime}}}_{R}^{\mathcal{I}}(\phi)\right)^{c}=\phi^{c}=U$ by Proposition 6.1 part (1).

2. Let $A \subseteq B$. Thus, $B^{c} \subseteq A^{c}$ and hence ${\overline{a p r^{\prime \prime}}}_{R}^{\mathcal{I}}\left(B^{c}\right) \subseteq{\overline{a p r^{\prime \prime}}}_{R}^{\mathcal{I}}\left(A^{c}\right)$ (by Proposition 6.1 part (2)). Then, $\left({\overline{a p r^{\prime \prime}}}_{R}^{\mathcal{I}}\left(A^{c}\right)\right)^{c} \subseteq\left({\overline{a p r^{\prime \prime}}}_{R}^{\mathcal{I}}\left(B^{c}\right)\right)^{c}$. So, $\underline{a p r}_{R}^{\prime \prime I}(A) \subseteq \underline{a p r}_{R}^{\prime \prime}(B)$.

3. Immediately by part (2).

4.

$$
\begin{aligned}
& {\underset{\operatorname{apr}}{ }}_{R}^{\prime \prime \mathcal{I}}(A \cap B)=\left({\overline{a p r^{\prime \prime}}}_{R}^{\mathcal{I}}(A \cap B)^{c}\right)^{c} . \\
& =\left(\overline{\operatorname{apr}}_{R}^{\prime \prime} \mathcal{I}\left(A^{c} \cup B^{c}\right)\right)^{c} \text {. } \\
& =\left({\overline{a p r^{\prime \prime}}}_{R}^{\mathcal{I}}\left(A^{c}\right) \cup{\overline{\operatorname{apr} r^{\prime \prime}}}_{R}^{\mathcal{I}}\left(B^{c}\right)\right)^{c}(\text { by No. (4) in Proposition 6.1). } \\
& =\left(\overline{\operatorname{apr}}_{R}^{\prime \prime} \mathcal{I}\left(A^{c}\right)\right)^{c} \cap\left({\overline{a p r^{\prime \prime}}}_{R}^{\mathcal{I}}\left(B^{c}\right)\right)^{c} \text {. } \\
& =\underline{\operatorname{apr}}_{R}^{\prime \prime \mathcal{I}}(A) \cap \underline{\operatorname{apr}}_{R}^{\prime \prime I}(B) \text {. }
\end{aligned}
$$

\section{Straightforward by Definition 6.1.}

6. Let $A^{c} \in \mathcal{I}$, then $\underline{\operatorname{apr}}_{R}^{\prime \prime I}(A)=\left({\overline{a p r^{\prime \prime}}}_{R}^{\mathcal{I}}\left(A^{c}\right)\right)^{c}=(\phi)^{c}=U$ by Proposition 6.1 part (6).

7. Let $\mathcal{I} \subseteq \mathcal{J}$. Then, $\overline{a p r^{\prime \prime}}{ }_{R}^{\mathcal{J}}\left(A^{c}\right) \subseteq{\overline{a p r^{\prime \prime}}}_{R}^{\mathcal{I}}\left(A^{c}\right)$ by Proposition 6.1 part (7). Thus, $\left(\overline{a p r^{\prime \prime}}{ }_{R}^{\mathcal{I}}\left(A^{c}\right)\right)^{c} \subseteq$

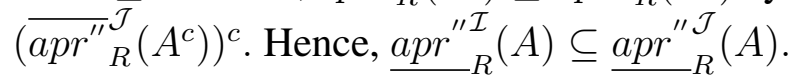

8. Let $\mathcal{I}=P(U)$, then $\underline{a p r}_{R}^{\prime \prime I}(A)=\left({\overline{a p r^{\prime \prime}}}_{R}^{\mathcal{I}}\left(A^{c}\right)\right)^{c}=(\phi)^{c}=U$ by Proposition 6.1 part (8).

9.

$$
\begin{aligned}
& {\operatorname{apr}_{R}}_{R}{ }^{\mathcal{I} \cap \mathcal{J}}(A)=\left({\overline{a p r^{\prime \prime}}}_{R}^{\mathcal{I} \cap \mathcal{J}}\left(A^{c}\right)\right)^{c} . \\
& =\left(\overline{\operatorname{apr}}_{R}{ }_{R}^{\mathcal{I}}\left(A^{c}\right) \cup{\overline{a p r^{\prime \prime}}}_{R}^{\mathcal{J}}\left(A^{c}\right)\right)^{c}(\text { by Proposition } 6.1 \text { part }(9)) \text {. } \\
& =\left({\overline{a p r^{\prime \prime}}}_{R}^{\mathcal{I}}\left(A^{c}\right)\right)^{c} \cap\left({\overline{a p r^{\prime \prime}}}_{R}^{\mathcal{J}}\left(A^{c}\right)\right)^{c} \text {. } \\
& =\underline{\operatorname{apr}}_{R}^{\prime \prime \mathcal{I}}(A) \cap \underline{\operatorname{apr}}_{R}^{\prime \prime} \mathcal{J}(A) \text {. }
\end{aligned}
$$


10.

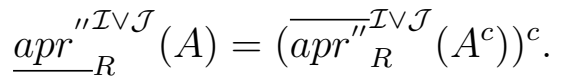

$$
\begin{aligned}
& =\left(\overline{\operatorname{apr}}_{R}{ }_{R}^{\mathcal{I}}\left(A^{c}\right) \cap \overline{\operatorname{apr}_{R}^{\prime \prime}}{ }_{R}^{\mathcal{J}}\left(A^{c}\right)\right)^{c}(\text { by Proposition } 6.1 \text { part }(10)) \text {. } \\
& =\left({\overline{a p r^{\prime \prime}}}_{R}^{\mathcal{I}}\left(A^{c}\right)\right)^{c} \cup\left(\overline{\operatorname{apr}_{R}^{\prime \prime}}{ }_{R}^{\mathcal{J}}\left(A^{c}\right)\right)^{c} \text {. } \\
& =\underline{a p r}_{R}^{\prime \prime \mathcal{I}}(A) \cup \underline{a p r}_{R}^{\prime \prime} \mathcal{J}(A) \text {. }
\end{aligned}
$$

Remark 6.1. 1. Example 3.1 (i) shows that the converse of part 2 in Propositions 6.1 and 6.2 is not necessarily to be true in general. Take

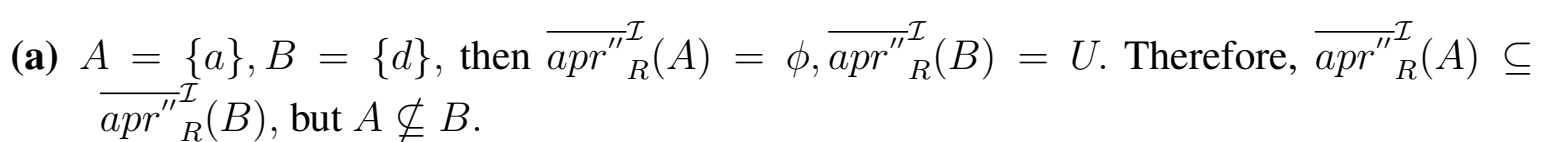

(b) $A=\{b\}, B=\{a, c, d\}$, then $\underline{\operatorname{apr}}_{R}^{\prime \prime \mathcal{I}}(A)=\phi, \underline{a p r}_{R}^{\prime \prime I}(B)=U$. Therefore, $\underline{a p r}_{R}^{\prime \prime I}(A) \subseteq$ $\underline{\operatorname{apr}}_{R}^{\prime \prime I}(B)$, but $A \nsubseteq B$.

2. Example 3.1 (ii) shows that the converse of parts 6, 7 and 8 in Propositions 6.1 and 6.2 is not necessarily to be true in general.

(i) For part 6 take

(a) $A=\{a, d\}$, then $\overline{a p r^{\prime \prime}}{ }_{R}^{\mathcal{J}}(A)=\phi$. Therefore, $\overline{\operatorname{apr}^{\prime \prime}}{ }_{R} \mathcal{J}(A)=\phi$, but $A \notin \mathcal{J}$.

(b) $A=\{b, c\}$, then $\underline{a p r}_{R}^{\prime \prime}(A)=U$. Therefore, $\underline{a p r}_{R}^{\prime \prime}(A)=U$, but $A^{c} \notin \mathcal{J}$.

(ii) For part 7 take (a) $A=\{a, d\}$, then ${\overline{a p r^{\prime \prime}}}_{R}^{\mathcal{I}}(A)=\{a\},{\overline{a p r^{\prime \prime}}}_{R}^{\mathcal{J}}(A)=\phi$. Therefore, $\overline{\operatorname{apr}}_{R}{ }_{R}^{\mathcal{J}}(A) \subseteq$
${\overline{a p r^{\prime \prime}}}_{R}^{\mathcal{I}}(A)$, but $\mathcal{I} \nsubseteq \mathcal{J}$.

(b) $A=\{b, c\}$, then $\underline{a p r}_{R}^{\prime \prime I}(A)=\{b, c, d\}, \underline{a p r}_{R}^{\prime \prime}(A)=U$. Therefore, $\underline{a p r}_{R}^{\prime \prime I}(A) \subseteq$ $\underline{\operatorname{apr}}_{R}^{\prime \prime}(A)$, but $\mathcal{I} \nsubseteq \mathcal{J}$.

(iii) For part 8 take

(a) $A=\{a, d\}$, then $\overline{a p r^{\prime \prime}}{ }_{R}^{\mathcal{J}}(A)=\phi$, but $\mathcal{J} \neq P(U)$.

(b) $A=\{b, c\}$, then $\underline{a p r}_{R}^{\prime \prime}(A)=U$, but $\mathcal{J} \neq P(U)$.

3. Example 3.1 (iii) shows that the inclusion of part 3 in Propositions 6.1 and 6.2 can not be replaced by equality relation in general. Take $A=\{a, d\}, B=\{b, c\}$, then

(a) ${\overline{a p r^{\prime \prime}}}_{R}^{\mathcal{I}}(A)={\overline{a p r^{\prime \prime}}}_{R}^{\mathcal{I}}(B)=U,{\overline{a p r^{\prime \prime}}}_{R}^{\mathcal{I}}(A \cap B)=\phi$. Therefore, ${\overline{a p r^{\prime \prime}}}_{R}^{\mathcal{I}}(A) \cap{\overline{a p r^{\prime \prime}}}_{R}^{\mathcal{I}}(B)=$ $U \neq \phi=\overline{\operatorname{apr}}_{R} \mathcal{I}(A \cap B)$.

(b) ${\frac{a p r^{\prime \prime I}}{R}}_{\phi \neq U}(A)=\frac{a p r^{\prime \prime I}}{R}(B)=\phi, \underline{a p r}_{R}^{\prime \prime I}(A \cup B)=U$. Therefore, $\underline{a p r}_{R}^{\prime \prime I}(A) \cup \underline{a p r}_{R}^{\prime \prime I}(B)=$ 
Remark 6.2. There are some properties which are not hold or satisfied in the fourth type.

(i) In Example 3.1 (i) take
1. $A=\{a\}$, then ${\overline{a p r^{\prime \prime}}}_{R}^{\mathcal{I}}(A)=\phi$. Hence, $A \nsubseteq{\overline{a p r^{\prime \prime}}}_{R}^{\mathcal{I}}(A)$.
2. $A=\{b, c, d\}$, then $\underline{a p r}_{R}^{\prime \prime I}(A)=U$. Hence, $\underline{a p r}_{R}^{\prime \prime I}(A) \nsubseteq A$.

(ii) In Example 3.1 (ii) take

1. $A=U$, then ${\overline{a p r^{\prime \prime}}}_{R}^{\mathcal{I}}(U)=\{a, b, c\}$. Hence, ${\overline{a p r^{\prime \prime}}}_{R}^{\mathcal{I}}(U) \neq U$.

2. $A=\phi$, then $\underline{\operatorname{apr}}_{R}^{\prime \prime I}(\phi)=\{d\}$. Hence, $\underline{\operatorname{apr}}_{R}^{\prime \prime I}(\phi) \neq \phi$.

The following theorem shows that the boundary of a subset decreases and the accuracy increases as the ideal on $U$ increases.

Theorem 6.1. Let $A \subseteq U, \mathcal{I}, \mathcal{J}$ be two ideals on $U$ and $R$ be a binary relation on $U$. If $\mathcal{I} \subseteq \mathcal{J}$. Then,
1. Boundary ${ }_{R}^{\prime \mathcal{J}}(A) \subseteq$ Boundary $_{R}^{\prime \prime}(A)$.

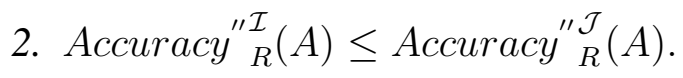

Proof. Similar to Theorem 3.1.

Remark 6.3. Example 3.1 (ii) shows that the converse of parts 1 and 2 in Theorem 6.1 is not necessary to be true in general. Take, $A=\{b, c\}$, then

1. Boundary ${ }_{R}^{\prime \mathcal{J}}(A)=\phi \subseteq \phi=$ Boundary $_{R}^{\prime \prime}(A)$, but $\mathcal{I} \nsubseteq \mathcal{J}$.

2. $\operatorname{Accuracy}_{R}^{\prime \prime \mathcal{I}}(A)=\frac{3}{2}<2=\operatorname{Accuracy}_{R}^{\prime \prime} \mathcal{J}(A)$, but $\mathcal{I} \nsubseteq \mathcal{J}$.

Theorem 6.2. Let $A \subseteq U, \mathcal{I}$ be an ideal on $U$ and $R_{1}, R_{2}$ be two similarity relations on $U$. If $R_{1} \subseteq R_{2}$, then
1. ${\overline{a p r^{\prime \prime}}}_{R_{1}}^{\mathcal{I}}(A) \subseteq{\overline{a p r^{\prime \prime}}}_{R_{2}}^{\mathcal{I}}(A)$.

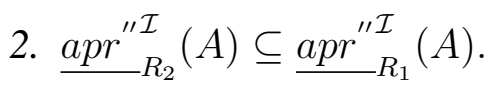
3. Boundary $_{R_{1}}^{\prime \prime \mathcal{I}}(A) \subseteq$ Boundary $_{R_{2}}^{\prime \prime I}(A)$.

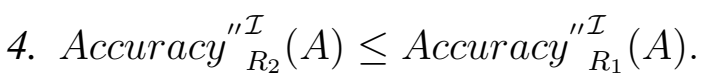

\section{Proof.}

1. Let $x \in{\overline{a p r^{\prime \prime}}}_{R_{1}}^{\mathcal{I}}(A)$. Then, $\exists y \in U$ such that $x \in<y>\breve{R}_{1} \cap A \notin \mathcal{I}$. Since, $<x>\breve{R}_{1} \subseteq<$ $x>\breve{R}_{2}$ (by Theorem 2.1 [8]). It follows that $x \in<y>\breve{R}_{2} \cap A \notin \mathcal{I}$. Thus, $x \in{\overline{a p r^{\prime \prime}}}_{R_{2}}^{\mathcal{I}}(A)$. Hence, ${\overline{a p r^{\prime \prime}}}_{R_{1}}^{\mathcal{I}}(A) \subseteq{\overline{a p r^{\prime \prime}}}_{R_{2}}^{\mathcal{I}}(A)$. 
2.

$$
\begin{aligned}
& x \in{\underset{\operatorname{apr}}{{ }^{\prime \prime}}}_{R_{2}}^{\mathcal{I}}(A)=\left({\overline{a p r^{\prime \prime}}}_{R_{2}}^{\mathcal{I}}\left(A^{c}\right)\right)^{c} . \\
& \subseteq\left({\overline{a p r^{\prime \prime}}}_{R_{1}}^{\mathcal{I}}\left(A^{c}\right)\right)^{c}(\text { by part }(1)) . \\
& =\underline{a p r}_{R_{1}}^{\prime I}(A) \text {. }
\end{aligned}
$$

3. Let $x \in$ Boundary $_{R_{1}}^{\prime I}(A)$. Then, $x \in{\overline{a p r^{\prime \prime}}}_{R_{1}}^{\mathcal{I}}(A)-\underline{a p r}_{R_{1}}^{\prime \prime I}(A)$. So, $x \in{\overline{a p r^{\prime \prime}}}_{R_{1}}^{\mathcal{I}}(A)$ and $x \in\left(\underline{a p r}_{R_{1}}^{\prime I}(A)\right)^{c}$. Thus, $x \in{\overline{a p r^{\prime \prime}}}_{R_{2}}^{\mathcal{I}}(A)$ and $x \in\left(\underline{a p r}_{R_{2}}^{\prime \prime} \mathcal{I}(A)\right)^{c}$ by parts 1 and 2 . Hence, $x \in$ Boundary $_{R_{2}}^{\prime \prime}(A)$. Therefore, Boundary ${ }_{R_{1}}^{\prime \mathcal{I}}(A) \subseteq$ Boundary $_{R_{2}}^{\prime \prime}(A)$.

4.

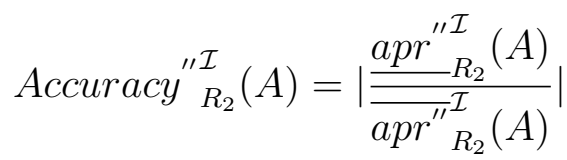

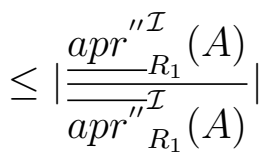

$$
\begin{aligned}
& =\text { Accuracy }_{R_{1}}^{\prime \prime}(A) \text {. }
\end{aligned}
$$

Remark 6.4. Example 3.3 shows that the inclusion and the less than in Theorem 6.2 can not be replaced by equality relation in general. Take

(i) $A=\{a, d\}$, then

$$
\begin{aligned}
& \text { 1. } \overline{\operatorname{apr}}_{{ }_{R_{1}}}^{\mathcal{I}}(A)=\{a, b\} \neq\{a, b, c\}={\overline{a p r^{\prime \prime}}}_{R_{2}}^{\mathcal{I}}(A) . \\
& \text { 2. } \operatorname{Accuracy}_{R_{1}}(A)=2 \neq \frac{4}{3}=\operatorname{Accuracy}_{R_{2}}(A) .
\end{aligned}
$$

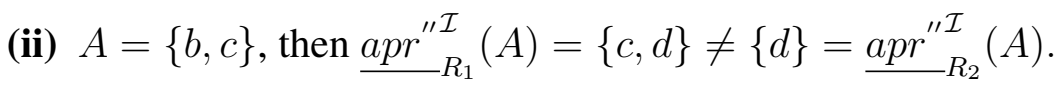

The following theorem presents the relationships between the current approximations in Definitions 3.1, 4.1, 5.1 and 6.1.

Theorem 6.3. Let $A \subseteq U, \mathcal{I}$ be an ideal on $U$ and $R$ be a reflexive relation on $U$. Then,

1. ${\underset{a p r}{\prime \prime}}_{R}^{\mathcal{I}}(A) \subseteq \underline{a p r}_{R}^{\mathcal{I}}(A) \subseteq \underline{a p r}_{R}^{\prime \mathcal{I}}(A) \subseteq A \subseteq \overline{a p r}_{R}^{\mathcal{I}}(A) \subseteq \overline{a p r}_{R}^{\mathcal{I}}(A) \subseteq{\overline{a p r^{\prime \prime}}}_{R}^{\mathcal{I}}(A)$.

2. Boundary $_{R}^{\prime \mathcal{I}}(A) \subseteq$ Boundary $_{R}^{\mathcal{I}}(A) \subseteq$ Boundary $_{R}^{\prime \prime}(A)$.

3. $\operatorname{Accuracy}_{R}^{\prime \mathcal{I}^{\mathcal{I}}}(A) \leq \operatorname{Accuracy}_{R}^{\mathcal{I}}(A) \leq \operatorname{Accuracy}_{R}^{\prime \mathcal{I}}(A)$. 
Proof. By Theorem 5.2, we have $\underline{a p r}_{R}^{\mathcal{I}}(A) \subseteq \underline{a p r}_{R}^{\prime \mathcal{I}}(A) \subseteq A \subseteq{\overline{a p r^{\prime}}}_{R}^{\mathcal{I}}(A) \subseteq \overline{a p r}_{R}^{\mathcal{I}}(A)$. To prove, $\underline{a p r}_{R}^{\prime \prime I}(A) \subseteq \operatorname{apr}_{R}^{\mathcal{I}}(A)$, let $x \in{\underline{a p r^{\prime \prime}}}_{R}^{\mathcal{I}}(A)=\overline{\overline{a p r}^{\prime \prime}}{ }_{R}^{\mathcal{I}}\left(A^{c}\right)^{c}$. Then, $x \notin{\overline{a p r^{\prime \prime}}}_{R}^{\mathcal{I}}\left(A^{c}\right)$. Thus, by

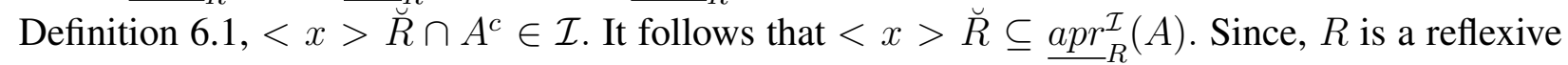
relation, then $x \in<x>\breve{R} \subseteq \underline{a p r}_{R}^{\mathcal{I}}(A)$. Therefore, $x \in \underline{a p r}_{R}^{\mathcal{I}}(A)$. To prove $\overline{\operatorname{apr}}_{R}^{\mathcal{I}}(A) \subseteq \overline{\operatorname{apr}}_{R}^{{ }^{\prime I}}(A)$, let $x \in \overline{\operatorname{apr}}_{R}^{\mathcal{I}}(A)$, then $\left\langle x>\breve{R} \cap A \notin \mathcal{I}\right.$. It follows that $<x>\breve{R} \subseteq \overline{\operatorname{apr}}^{{ }^{\prime \prime}}{ }_{R}(A)$. Since, $R$ is a reflexive relation, then $x \in<x>\breve{R} \subseteq{\overline{a p r^{\prime \prime}}}_{R}^{\mathcal{I}}(A)$. Therefore, $x \in{\overline{a p r^{\prime \prime}}}_{R}^{\overline{\mathcal{I}}}(A)$.

Remark 6.5. Example 3.1 (iii) shows that the inclusion and the less than in Theorem 6.3 can not be replaced by equality relation in general. Take $A=\{b, c\}$, then $\overline{a p r}_{R}^{\mathcal{I}}(A)=\{a, b, c\} \varsubsetneqq U=$ ${\overline{a p r^{\prime \prime}}}_{R}^{\mathcal{I}}(A)$. Moreover, Take $A=\{a, d\}$, then

1. $\underline{\operatorname{apr}}_{R}^{\prime \prime}{ }_{R}^{\mathcal{I}}(A)=\phi \varsubsetneqq\{d\}=\underline{a p r}_{R}^{\mathcal{I}}(A)$.

2. Boundary $_{R}^{\mathcal{I}}(A)=\{a, b, c\} \varsubsetneqq U=$ Boundary $_{R}^{\prime \prime I}(A)$.

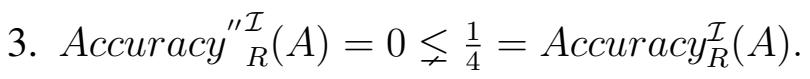

Remark 6.6. From the above results, it is noted that there are different methods to approximate the sets. The best of these methods is the third type in Section 5, since the boundary regions in this case are decreased (or canceled) by increasing the lower approximations and decreasing the upper approximations with the comparison of the other types in the other sections. Moreover, the accuracy is more accurate than the other types.

The following theorem presents the relationships between the current approximations in Definition 6.1 and the previous one in Definition 2.6 [8].

Theorem 6.4. Let $A \subseteq U, \mathcal{I}$ be an ideal on $U$ and $R$ be a similarity relation on a nonempty set U. Then,

1. $\overline{\operatorname{apr}}_{R} \mathcal{I}(A) \subseteq \overline{\operatorname{apr}}_{R}^{\prime \prime}(A)$.

2. $\underline{\operatorname{apr}}_{R}^{\prime \prime}(A) \subseteq \underline{\operatorname{apr}}_{R}^{\prime \prime I}(A)$.

3. Boundary $_{R}^{\prime I}(A) \subseteq$ Boundary $_{R}{ }_{R}(A)$.

4. $\operatorname{Accuracy}_{R}{ }_{R}(A) \leq \operatorname{Accuracy}_{R}^{\prime \prime}(A)$.

Proof. The proof is similar to that of Theorem 3.3.

Remark 6.7. Example 3.3 shows that the inclusion and the less than in Theorem 6.4 can not be replaced by equality relation in general. Take $A=\{a, c, d\}$, then

1. $\overline{a p r^{\prime \prime}}{ }_{R_{1}}^{\mathcal{I}}(A)=\{a, b, d\} \neq U=\overline{a p r^{\prime \prime}}{ }_{R_{1}}(A)$.

2. ${\underbrace{a p r}}_{R_{1}}^{\prime \prime}(A)=\{c, d\} \neq U=\underline{a p r}_{R_{1}}^{\prime \mathcal{I}}(A)$. 
3. Boundary ${ }_{R_{1}}^{\prime I}(A)=\phi \neq\{a, b\}=$ Boundary $_{R_{1}}(A)$.

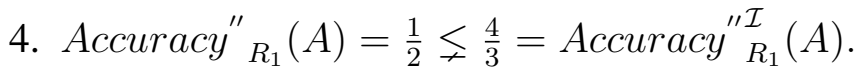

\section{Medical applications}

Example 7.1. A medical application in decision-making of COVID-19

The purpose of this example is to demonstrate the significance of the current approximations in obtaining the best tools to identify deciding factors of infections for COVID-19 in humans. Information in the Table 1 has been collected by the World Health Organization as well as through medical groups specializing in corona virus (COVID-19) [11]. It is from 1000 patients and reduced to 10 patients as the attributes in rows (objects) are identical. So, the set of objects is $U=\left\{p_{1}, p_{2}, p_{3}, p_{4}, p_{5}, p_{6}, p_{7}, p_{8}, p_{9}, p_{10}\right\}$. The most symptoms (set of attributes) of COVID-19 are $=\left\{\right.$ Difficulty breathing $=a_{1}$, Chest pain $=a_{2}$, Headache $=a_{3}$, Dry cough $=a_{4}$, High Temperature $a_{5}$, Loss of smell or taste $\left.=a_{6}\right\}$ and Decision COVID-19 $=\{d\}$, as shown Table 1 .

Table 1: The information's decisions data set.

\begin{tabular}{|c|c|c|c|c|c|c|c|}
\hline Patients & \multicolumn{2}{|c|}{ Serious symptoms } & \multicolumn{4}{|c|}{ Most common symptoms } & \multirow{2}{*}{$\begin{array}{c}\text { Decision } \\
\text { COVID }-19 \\
\end{array}$} \\
\hline & Difficulty breathing $=a_{1}$ & Chest pain $=a_{2}$ & Headache $=a_{3}$ & Dry cough $=a_{4}$ & High Temperature $=a_{5}$ & Loss of taste or smell $=a_{6}$ & \\
\hline$\left\{p_{1}\right\}$ & yes & yes & no & yes & yes & yes & yes \\
\hline$\left\{p_{2}\right\}$ & yes & yes & yes & yes & yes & yes & yes \\
\hline$\left\{p_{3}\right\}$ & yes & yes & no & yes & no & yes & no \\
\hline$\left\{p_{4}\right\}$ & yes & yes & no & no & no & no & no \\
\hline$\left\{p_{5}\right\}$ & yes & yes & no & yes & no & no & no \\
\hline$\left\{p_{6}\right\}$ & yes & no & yes & yes & yes & no & yes \\
\hline$\left\{p_{7}\right\}$ & no & no & no & yes & yes & no & yes \\
\hline$\left\{p_{8}\right\}$ & no & no & no & yes & yes & no & no \\
\hline$\left\{p_{9}\right\}$ & no & no & no & no & no & yes & yes \\
\hline$\left\{p_{10}\right\}$ & no & no & yes & yes & yes & no & yes \\
\hline
\end{tabular}

From Table 1, the symptoms are given as follows: $\mathcal{V}\left(p_{1}\right)=\left\{a_{1}, a_{2}, a_{4}, a_{5}, a_{6}\right\}, \mathcal{V}\left(p_{2}\right)=$ $\left\{a_{1}, a_{2}, a_{3}, a_{4}, a_{5}, a_{6}\right\}, \mathcal{V}\left(p_{3}\right)=\left\{a_{1}, a_{2}, a_{4}, a_{6}\right\}, \mathcal{V}\left(p_{4}\right)=\left\{a_{1}, a_{2}\right\}, \mathcal{V}\left(p_{5}\right)=\left\{a_{1}, a_{2}, a_{4}\right\}, \mathcal{V}\left(p_{6}\right)=$ $\left\{a_{1}, a_{3}, a_{4}, a_{5}\right\}, \mathcal{V}\left(p_{7}\right)=\mathcal{V}\left(p_{8}\right)=\left\{a_{4}, a_{5}\right\}, \mathcal{V}\left(p_{9}\right)=\left\{a_{6}\right\}$ and $\mathcal{V}\left(p_{10}\right)=\left\{a_{3}, a_{4}, a_{5}\right\}$. Hence, the similarity relation is given as follows:

$p_{i} \mathcal{R} p_{j} \Leftrightarrow \mathcal{V}\left(p_{i}\right) \cap \mathcal{V}\left(p_{j}\right) \neq \phi$. Consequently, $\mathcal{R}=\Delta \cup\left\{\left(p_{1}, p_{2}\right),\left(p_{1}, p_{3}\right),\left(p_{1}, p_{4}\right),\left(p_{1}, p_{5}\right),\left(p_{1}, p_{6}\right)\right.$, $\left(p_{1}, p_{7}\right),\left(p_{1}, p_{8}\right),\left(p_{1}, p_{9}\right),\left(p_{1}, p_{10}\right),\left(p_{2}, p_{1}\right),\left(p_{2}, p_{3}\right),\left(p_{2}, p_{4}\right),\left(p_{2}, p_{5}\right),\left(p_{2}, p_{6}\right),\left(p_{2}, p_{7}\right),\left(p_{2}, p_{8}\right)$, $\left(p_{2}, p_{9}\right),\left(p_{2}, p_{10}\right),\left(p_{3}, p_{1}\right),\left(p_{3}, p_{2}\right),\left(p_{3}, p_{4}\right),\left(p_{3}, p_{5}\right),\left(p_{3}, p_{6}\right),\left(p_{3}, p_{7}\right),\left(p_{3}, p_{8}\right),\left(p_{3}, p_{9}\right),\left(p_{3}, p_{10}\right)$, $\left(p_{4}, p_{1}\right),\left(p_{4}, p_{2}\right),\left(p_{4}, p_{3}\right),\left(p_{4}, p_{5}\right),\left(p_{4}, p_{6}\right),\left(p_{5}, p_{1}\right),\left(p_{5}, p_{2}\right),\left(p_{5}, p_{3}\right),\left(p_{5}, p_{4}\right),\left(p_{5}, p_{6}\right),\left(p_{5}, p_{7}\right)$, $\left(p_{5}, p_{8}\right),\left(p_{5}, p_{10}\right),\left(p_{6}, p_{1}\right),\left(p_{6}, p_{2}\right),\left(p_{6}, p_{3}\right),\left(p_{6}, p_{4}\right),\left(p_{6}, p_{5}\right),\left(p_{6}, p_{7}\right),\left(p_{6}, p_{8}\right),\left(p_{6}, p_{10}\right),\left(p_{7}, p_{1}\right)$, $\left(p_{7}, p_{2}\right),\left(p_{7}, p_{3}\right),\left(p_{7}, p_{5}\right),\left(p_{7}, p_{6}\right),\left(p_{7}, p_{8}\right),\left(p_{7}, p_{10}\right),\left(p_{8}, p_{1}\right),\left(p_{8}, p_{2}\right),\left(p_{8}, p_{3}\right),\left(p_{8}, p_{5}\right),\left(p_{8}, p_{6}\right)$, $\left(p_{8}, p_{7}\right),\left(p_{8}, p_{10}\right),\left(p_{9}, p_{1}\right),\left(p_{9}, p_{2}\right),\left(p_{9}, p_{3}\right),\left(p_{10}, p_{1}\right),\left(p_{10}, p_{2}\right),\left(p_{10}, p_{3}\right),\left(p_{10}, p_{5}\right),\left(p_{10}, p_{6}\right)$, $\left.\left(p_{10}, p_{7}\right),\left(p_{10}, p_{8}\right)\right\}$, thus $<p_{1}>\breve{\mathcal{R}}=<p_{2}>\breve{\mathcal{R}}=<p_{3}>\widetilde{\mathcal{R}}=<p_{4}>\breve{\mathcal{R}}=<p_{5}>\breve{\mathcal{R}}=$ 
$<p_{6}>\breve{\mathcal{R}}=<p_{7}>\breve{\mathcal{R}}=<p_{8}>\breve{\mathcal{R}}=<p_{9}>\breve{\mathcal{R}}=<p_{10}>\breve{\mathcal{R}}=U$. Let $\mathcal{I}=\left\{\phi,\left\{p_{3}\right\},\left\{p_{4}\right\}\right.$,

$\left\{p_{5}\right\},\left\{p_{8}\right\},\left\{p_{3}, p_{4}\right\},\left\{p_{3}, p_{5}\right\},\left\{p_{3}, p_{8}\right\},\left\{p_{4}, p_{5}\right\},\left\{p_{4}, p_{8}\right\},\left\{p_{5}, p_{8}\right\},\left\{p_{3}, p_{4}, p_{5}\right\},\left\{p_{3}, p_{4}, p_{8}\right\}$,

$\left.\left\{p_{3}, p_{5}, p_{8}\right\},\left\{p_{4}, p_{5}, p_{8}\right\},\left\{p_{3}, p_{4}, p_{5}, p_{8}\right\}\right\}$.

Hence, Table 1 represents a decision system and thus the patients with confirmed infections for COVID-19 are surely $A=\left\{p_{1}, p_{2}, p_{6}, p_{7}, p_{9}, p_{10}\right\}$. Then

1. by the previous approximations [8], the first (second/third) kind of the lower and upper approximations, boundary regions and accuracy of $A$ is respectively $\phi, U, U, 0$. This means that the patients $p_{1}, p_{2}, p_{6}, p_{7}, p_{9}$ and $p_{10}$ are not infections for COVID-19, which contradicts to the decision system in Table 1 . Therefore, we are unable to decide whether the patient is infected for COVID-19 and this produced vagueness in decision making regarding the medical diagnosis. Consequently, Dai et al.'s methods [8] are not suitable for obtaining an accurate decision.

2. by the present second kind of the lower and upper approximations, boundary regions and accuracy of $A$ is respectively $A, U,\left\{p_{3}, p_{4}, p_{5}, p_{8}\right\}, \frac{6}{10}$. This means that the patients $p_{1}, p_{2}, p_{6}, p_{7}, p_{9}$ and $p_{10}$ are surely infected for COVID-19 according to the present technique which is coincided with Table 1. Accordingly, the vagueness is reduced in the data and the accuracy measure is increased.

Example 7.2. Medical application in decision-making of the heart attacks problem

In this example the proposed methods in decision making to heart attacks are applied. The data set in Table 2 is carried out at Al-Azhar University's cardiology department [7] (Hospital of Sayed Glal University, Cairo, Egypt). Table 2 represents the set of objects (patients) $U=$ $\left\{p_{1}, p_{2}, p_{3}, p_{4}, p_{5}, p_{6}, p_{7}, p_{8}, p_{9}, p_{10}, p_{11}, p_{12}\right\}$ and reduced to $\left\{p_{1}, p_{2}, p_{3}, p_{4}, p_{5}, p_{8}, p_{9}\right\}$ as the attributes in rows (objects) are identical. The study is included patients with different symptoms (the set of attributes $)=\left\{\right.$ detailed history $=a_{1}$, physical examination $=a_{2}$, full labs $=a_{3}$, resting $\mathrm{ECG}=a_{4}$, conventional echo assessment $\left.=a_{5}\right\}$ and Decision of heart attacks is confirmed or ruled out $=\{d\}$ as shown in Table 2 .

Table 2: The information's decisions data set.

\begin{tabular}{|c||c|c|c|c|c||c|}
\hline Patients & breathlessness $=a_{1}$ & orthopnea $=a_{2}$ & paroxysmal nocturnal dyspnea $=a_{3}$ & reduced exercise tolerance $=a_{4}$ & ankle swelling $=a_{5}$ & $d$ \\
\hline \hline$\left\{p_{1}\right\}$ & yes & yes & yes & yes & no & yes \\
\hline$\left\{p_{2}\right\}$ & no & no & no & yes & yes \\
\hline$\left\{p_{3}\right\}$ & yes & yes & yes & yes & no \\
\hline$\left\{p_{4}\right\}$ & no & no & no & yes & no \\
\hline$\left\{p_{5}\right\}$ & yes & no & no & yes & no \\
\hline$\left\{p_{8}\right\}$ & yes & yes & no & yes & yes & no \\
\hline$\left\{p_{9}\right\}$ & yes & no & yes & yes & yes & yes \\
\hline
\end{tabular}


Therefore, from Table 2, the symptoms are given as follows: $\mathcal{V}\left(p_{1}\right)=\left\{a_{1}, a_{2}, a_{3}, a_{4}\right\}, \mathcal{V}\left(p_{2}\right)=$ $\left\{a_{4}, a_{5}\right\}, \mathcal{V}\left(p_{3}\right)=\left\{a_{1}, a_{2}, a_{3}, a_{4}, a_{5}\right\}, \mathcal{V}\left(p_{4}\right)=\left\{a_{4}\right\}, \mathcal{V}\left(p_{5}\right)=\left\{a_{1}, a_{4}, a_{5}\right\}, \mathcal{V}\left(p_{8}\right)=\left\{a_{1}, a_{2}, a_{4}, a_{5}\right\}$ and $\mathcal{V}\left(p_{9}\right)=\left\{a_{1}, a_{3}, a_{4}\right\}$. Hence, the following binary relation is obtained:

$p_{i} \mathcal{R} p_{j} \Leftrightarrow \mathcal{V}\left(p_{i}\right) \subsetneq \mathcal{V}\left(p_{j}\right)$. Consequently, $\mathcal{R}=\left\{\left(p_{1}, p_{3}\right),\left(p_{2}, p_{3}\right),\left(p_{2}, p_{5}\right),\left(p_{2}, p_{8}\right),\left(p_{4}, p_{1}\right),\left(p_{4}, p_{2}\right)\right.$, $\left.\left(p_{4}, p_{3}\right),\left(p_{4}, p_{5}\right),\left(p_{4}, p_{8}\right),\left(p_{4}, p_{9}\right),\left(p_{5}, p_{3}\right),\left(p_{5}, p_{8}\right),\left(p_{6}, p_{1}\right)\left(p_{8}, p_{3}\right),\left(p_{9}, p_{1}\right),\left(p_{9}, p_{3}\right)\right\}$, thus $<p_{1}>$ $\breve{\mathcal{R}}=<p_{2}>\widetilde{\mathcal{R}}=<p_{3}>\breve{\mathcal{R}}=<p_{5}>\breve{\mathcal{R}}=<p_{8}>\breve{\mathcal{R}}=<p_{9}>\breve{\mathcal{R}}=\left\{p_{1}, p_{2}, p_{3}, p_{5}, p_{8}, p_{9}\right\},<$ $p_{4}>\breve{\mathcal{R}}=\phi$. Let $\mathcal{I}=\left\{\phi,\left\{p_{2}\right\},\left\{p_{4}\right\},\left\{p_{5}\right\},\left\{p_{2}, p_{4}\right\},\left\{p_{2}, p_{5}\right\},\left\{p_{4}, p_{5}\right\},\left\{p_{2}, p_{4}, p_{5}\right\}\right\}$.

Thus, Table 2 represents a decision system and thus the patients with confirmed heart attacks were surely $A=\left\{p_{1}, p_{3}, p_{8}, p_{9}\right\}$. Thus, we computed the approximations, the boundary and the accuracy measure of $A$ respectively using

1. the first (second/third) kind in Dai et al.'s approach [8] are $\left\{p_{4}\right\},\left\{p_{1}, p_{2}, p_{3}, p_{5}, p_{8}, p_{9}\right\}$, $\left\{p_{1}, p_{2}, p_{3}, p_{5}, p_{8}, p_{9}\right\}, \frac{1}{6}$ which means that $A$ is a rough set according to the Dai technique. Further, the patients $p_{4}$ and $p_{6}$ experience heart attacks, which contradict the decision system in Table 2. Therefore, we are unable to decide whether the patient is experiencing heart attacks.

2. the second kind in present approach are $A,\left\{p_{1}, p_{2}, p_{3}, p_{5}, p_{8}, p_{9}\right\},\left\{p_{2}, p_{5}\right\}, \frac{4}{6}$. This means the patients $\left\{p_{1}, p_{3}, p_{8}, p_{9}\right\}$ surely experienced heart attacks according to the proposed technique which is coincided with Table 2 . Additionally, the boundary region is reduced and the accuracy measure is increased.

Remark 7.1. It should be noted that

1. the relation is identified according to the viewpoint of the system's experts.

2. the Dai et al.'s approximations [8] satisfied some properties of the basic properties of rough set when the relation is binary relation. Meanwhile, the reminders of the properties are achieved only if the relation is a similarity relation.

3. the present techniques are extended the applications fields of the rough sets. As, the similarity relations do not always hold in many real-life applications and consequently this restriction limits the wide applications of this set.

\section{Conclusions}

Rough set theory is a new mathematical tool to deal with vagueness and imperfect knowledge by using the lower and upper approximations. Whereas, ideal is a fundamental concept in topological spaces and played an important role in the study of a generalization of rough set. Since the advent of the ideals, several research papers with interesting results in different respects came to existence. In view of the recent applications of ideals in the rough set theory, it seems very natural to extend the interesting concept of rough set further by using ideals that mainly did here. So, in this paper different methods depended on ideals and the maximal right neighborhood which 
generated by binary relations are proposed to approximate the sets. The use of ideals made the boundary region reduced and consequently, the accuracy measure was higher than the use of Dai et al.'s approximations [8] which depended only on the maximal neighborhood which generated by similarity relations. Hence, the present approach was extension of Dai et al.'s approach [8]. The basic properties of the current methods were studied. More importantly, it was proved for three of the current methods that the corresponding lower and upper approximations, boundary regions, accuracy measures and roughness measures were monotonic. Moreover, to add strength and make the current work vivid two medical applications were proposed to illustrate the main idea of the present results. The present techniques were successful and powerful techniques to reduce the boundary region and improve the accuracy measure. Therefore, this helped the medical staff to make an accurate decision about the diagnosis of patients.

In the upcoming works, we will

1. study new types of approximations induced from neighborhoods and ideals.

2. search how these approximations can be applied to model real-life issues.

3. investigate the concepts and results presented, herein, in generalize rough multisets via multisets ideals.

\section{Compliance with ethical standards}

Conflict of interest: The author declares that they have no conflict of interest.

Ethical approval: This article does not contain any studies with human participants or animals performed by any of the author.

\section{References}

[1] E. A. Abo-Tabl, A comparison of two kinds of definitions of rough approximations based on a similarity relation, Inform. Sci. 181 (2011) 2587-2596.

[2] H. M. Abu-Doniaa, Comparison between different kinds of approximations by using a family of binary relations, Knowl Based Syst. 21 (2008) 911-919.

[3] H. M. Abu-Doniaa, Multi knowledge based rough approximations and applications, Knowl Based Syst. 26 (2012) 20-29.

[4] A. A. Allam, M. Y. Bakeir, E. A. Abo-Tabl, New approach for basic rough set concepts, in: International Workshop on Rough Sets, Fuzzy Sets, Data Mining, and Granular Computing. Lecture Notes in Artificial Intelligence, 3641, Springer, Regina, 2005, pp. 64-73.

[5] A. A. Allam, M. Y. Bakeir, E. A. Abo-Tabl, New approach for closure spaces by relations, Acta Mathematica Academiae Paedagogicae Nyiregyháziensis 22 (2006) 285-304. 
[6] T. M. Al-shami, H. Işık, A. S. Nawar, R. A. Hosny, Some topological approaches for generalized rough sets via ideals, Mathematical Problems in Engineering, https://doi.org/10.1155/2021/5642982 (2021).

[7] A. A. Azzam, A. M. Khalil, S. G. Li, Medical applications via minimal topological structure, J. Intell. Fuzzy Syst. 39 (2020) 47234730.

[8] J. Dai, S. Gao, G. Zheng, Generalized rough set models determined by multiple neighborhoods generated from a similarity relation, Soft Computing 13 (2018) 2081-2094.

[9] J. Dai, W. Wang, Q. Xu, H. Tian, Uncertainty measurement for interval-valued decision systems based on extended conditional entropy, Knowl Based Syst. 27 (2012) 443-450.

[10] J. Dai, Q. Xu, Approximations and uncertainty measures in incomplete information systems, Inform. Sci. 198 (2012) 62-80.

[11] M. A. El Safty, S. AlZahrani, Topological modeling for symptom reduction of Corona virus, Punjab Uni. J. Math. 53 (2021) 4759.

[12] M. Hosny, Topological approach for rough sets by using J-nearly concepts via ideals, Filomat 34 (2020) 273-286.

[13] M. Hosny, Idealization of $j$-approximation spaces, Filomat 34 (2020) 287-301.

[14] M. Hosny, Rough sets theory via new topological notions based on ideals and applications, AIMS Mathematics 7 (2021) 869-902.

[15] R. A. Hosny, B. A. Asaad, A. A. Azzam, T. M. Al-Shami, Various topologies generated from $E_{j}$-neighbourhoods via ideals, Complexity, https://doi.org/10.1155/2021/4149368 (2021).

[16] D. Jankovic, T. R. Hamlet, New topologies from old via ideals, Amer. Math. Monthly 97 (1990) 295-310.

[17] J. Järinen, Approximations and rough sets based on tolerances, in: W. Ziarko, Y. Yao (Eds.), Lecture Notes in Artifical Intelligence 2475(RSCTC 2002), Springer, Berlin (2002) $123-130$.

[18] A. Kandil, S. A. El-Sheikh, M. Hosny, M. Raafat, Bi-ideal approximation spaces and their applications, Soft Computing, https://doi.org/10.1007/s00500-020-04720-2 (2020).

[19] M. Kondo, On the structure of generalized rough sets, Inform. Sci. 176 (2005) 589-600.

[20] A. M. Kozae, On topology expansions by ideals and applications, Chaos, Solitons and Fractals 13 (2002) 55-60.

[21] M. Kryszkiewicz, Rough set approach to incomplete information systems, Inform. Sci. 112 (1998) 39-49. 
[22] K. Kuratowski, Topology Vol. I, Academic Press, New York, 1966.

[23] J. Nieminen, Rough tolerance equality, Fund. Inform. 11 (1988) 289-296.

[24] Z. Pawlak, Rough sets, Int. J. of Information and Computer Sciences 11 (1982) 341-356.

[25] Z. Pawlak, Rough concept analysis, Bull. Pol. Acad. Sci. Math. 33 (1985) 9-10.

[26] D. Pei, Z. Xu, Transformation of rough set models, Knowl Based Syst. 20 (2007) 745-751.

[27] J. A. Pomykala, About tolerance and similarity relations in information systems, in: J. Alpigini, J. Peters, A. Skowron, N. Zhong (Eds.), Lecture Notes in Artificial Intelligence 2475 (RSCTC 2002), Springer, Berlin (2002) 175-182.

[28] A. Skowron, J. Stepaniuk, Tolerance approximation spaces, Fund. Inform. 27 (1996) $245-253$.

[29] A. Skowron, D. Vanderpooten, A generalized definition of rough approximations based on similarity, IEEE Trans. Knowl. Data Eng. 12 (2000) 331-336.

[30] R. Slowinski, J. Stefanowski, Rough-set reasoning about uncertain data, Fund. Inform. 27 (1996) 229-243.

[31] R. Vaidynathaswamy, The localization theory in set topology, Proc. Ind. Acad. of Sci. 20 (1945) 515-61.

[32] Y.Y. Yao, Two views of the theory of rough sets in finite universes, Int. J. Approximate Reason. 15 (1996) 291-317.

[33] Y.Y. Yao, in: L. Polkowski, A. Skowron (Eds.), Generalized rough set models, Rough sets in knowledge Discovery 1, Physica Verlag, Heidelberg, 1998, pp. 286-318.

[34] Y. Y. Yao, Constructive and algebraic methods of the theory of rough sets, Inform. Sci. 109 (1998) 21-47.

[35] Y. Y. Yao, On generalized Pawlak approximation operators, Rough Sets and Current Trends in Comput. LNAI 1424 (1998) 298-307.

[36] Y. Y. Yao, Relational interpretations of neighborhood operators and rough set approximation operators, Inform. Sci. 1119 (1998) 239-259.

[37] X. Zhang, J. Dai, Y. Yu, On the union and intersection operations of rough sets based on various approximation spaces, Inform. Sci. 292 (2015) 214-229.

[38] W. Zhu, Generalized rough sets based on relations, Inform. Sci. 177 (2007) 4997-5011. 